R. Okazaki and K. Yanagawa

Nagoya Math. J.

Vol. 196 (2009), 87-116

\title{
DUALIZING COMPLEX OF A TORIC FACE RING
}

\author{
RYOTA OKAZAKI AND KOHJI YANAGAWA
}

\begin{abstract}
A toric face ring, which generalizes both Stanley-Reisner rings and affine semigroup rings, is studied by Bruns, Römer and their coauthors recently. In this paper, under the "normality" assumption, we describe a dualizing complex of a toric face ring $R$ in a very concise way. Since $R$ is not a graded ring in general, the proof is not straightforward. We also develop the squarefree module theory over $R$, and show that the Cohen-Macaulay, Buchsbaum, and Gorenstein* properties of $R$ are topological properties of its associated cell complex.
\end{abstract}

\section{$\S 1$. Introduction}

Stanley-Reisner rings and (normal) affine semigroup rings are important subjects of combinatorial commutative algebra. The notion of toric face rings, which originated in Stanley [12], generalizes both of them, and has been studied by Bruns, Römer, and their coauthors recently (e.g. [2], [5], [8]). Contrary to Stanley-Reisner rings and affine semigroup rings, a toric face ring does not admit a nice multi-grading in general. So, even if the results can be easily imagined from these classical examples, the proofs sometimes require technical argument.

Now we start the definition of a toric face ring. Let $\mathcal{X}$ be a finite cell complex with $\emptyset \in \mathcal{X}$. Assume that the closure $\bar{\sigma}$ of each $i$-cell $\sigma \in \mathcal{X}$ is homeomorphic to an $i$-dimensional ball, and for given two cells $\sigma, \tau \in \mathcal{X}$ there exists $v \in \mathcal{X}$ with $\bar{\sigma} \cap \bar{\tau}=\bar{v}$ (we allow the case $v=\emptyset$ ). A simplicial complex and the cell complex associated with a polytope are examples of our $\mathcal{X}$.

We assign a pointed polyhedral cone $C_{\sigma} \subset \mathbb{R}^{d_{\sigma}}$ to each $\sigma \in \mathcal{X}$ so that the following condition is satisfied. (We say a cone is pointed if it contains no line.)

Received September 10, 2008

Accepted April 30, 2009.

2000 Mathematics Subject Classification: Primary 13F55, 13 D25.

The second author is partially supported by Grant-in-Aid for Scientific Research (c) (no. 19540028). 
(*) $\operatorname{dim} C_{\sigma}=\operatorname{dim} \sigma+1$, and there is a one-to-one correspondence between ffaces of $\left.C_{\sigma}\right\}$ and $\{\tau \in \mathcal{X} \mid \tau \subset \bar{\sigma}\}$. The face of $C_{\sigma}$ corresponding to $\tau$ is isomorphic to $C_{\tau}$ by a map $\iota_{\sigma, \tau}: C_{\tau} \rightarrow C_{\sigma}$. These maps satisfy $\iota_{\sigma, \sigma}=\operatorname{id}_{C_{\sigma}}$ and $\iota_{\sigma, \tau} \circ \iota_{\tau, v}=\iota_{\sigma, v}$ for all $\sigma, \tau, v \in \mathcal{X}$ with $\bar{\sigma} \supset \bar{\tau} \supset$.

For example, a pointed fan (i.e., a fan consisting of pointed cones) gives such a structure. Here $\iota_{\sigma, \tau}$ 's are inclusion maps, and $\mathcal{X}$ is a "cross-section" of the fan.

Next we define a monoidal complex $\mathcal{M}$ supported by $\left\{C_{\sigma}\right\}_{\sigma \in \mathcal{X}}$ as follows.

(**) To each $\sigma \in \mathcal{X}$, we assign a finitely generated additive submonoid $\mathbf{M}_{\sigma} \subset\left(\mathbb{Z}^{d_{\sigma}} \cap C_{\sigma}\right) \subset \mathbb{R}^{d_{\sigma}}$ with $\mathbb{R}_{>0} \mathbf{M}_{\sigma}=C_{\sigma}$. For $\sigma, \tau \in \mathcal{X}$ with $\bar{\sigma} \supset \tau$, the map $\iota_{\sigma, \tau}: C_{\tau} \rightarrow C_{\sigma}$ induces an isomorphism $\mathbf{M}_{\tau} \cong \mathbf{M}_{\sigma} \cap \iota_{\sigma, \tau}\left(C_{\tau}\right)$ of monoids.

If $\Sigma$ is a rational pointed fan in $\mathbb{R}^{n}$, then $\left\{\mathbb{Z}^{n} \cap C\right\}_{C \in \Sigma}$ gives a monoidal complex.

For a monoidal complex $\mathcal{M}$ on a cell complex $\mathcal{X}$, we set $|\mathcal{M}|:=$ $\lim _{\sigma \in \mathcal{X}} \mathbf{M}_{\sigma}$, where the direct limit is taken with respect to $\iota_{\sigma, \tau}: \mathbf{M}_{\tau} \rightarrow \mathbf{M}_{\sigma}$ for $\sigma, \tau \in \mathcal{X}$ with $\bar{\sigma} \supset \tau$. If $\mathcal{M}$ comes from a fan in $\mathbb{R}^{n}$, then $|\mathcal{M}|$ can be identified with $\bigcup_{\sigma \in \mathcal{X}} \mathbf{M}_{\sigma} \subset \mathbb{Z}^{n}$. The $\mathbb{k}$-vector space

$$
\mathbb{k}[\mathcal{M}]:=\bigoplus_{a \in|\mathcal{M}|} \mathbb{k} t^{a}
$$

with the multiplication

$$
t^{a} \cdot t^{b}= \begin{cases}t^{a+b} & \text { if } a, b \in \mathbf{M}_{\sigma} \text { for some } \sigma \in \mathcal{X} \\ 0 & \text { otherwise }\end{cases}
$$

has a $\mathbb{k}$-algebra structure. We call $\mathbb{k}[\mathcal{M}]$ the toric face ring of $\mathcal{M}$. If $\mathcal{M}$ comes from a fan in $\mathbb{R}^{n}$, then $\mathbb{k}[\mathcal{M}]$ has a natural $\mathbb{Z}^{n}$-grading. However, this is not true in general (cf. Example 2.9 below).

ExAmple 1.1. (1) Let $\Delta$ be a simplicial complex. Attaching the monoid $\mathbb{N}^{i+1}$ to each $i$-dimensional face of $\Delta$, we get a monoidal complex $\mathcal{M}$ on $\Delta$. In this case, $\mathbb{k}[\mathcal{M}]$ coincides with the Stanley-Reisner ring $\mathbb{k}[\Delta]$. An affine semigroup ring is also a toric face ring corresponding to the case when $\mathcal{X}$ has a unique maximal cell. 
(2) Let $\mathcal{X}$ be a two-dimensional cell complex given by the boundary of a cube. Assigning normal semigroup rings of the form $\mathbb{k}[x, y, z, w] /(x z-y w)$ to all two-dimensional cells, we get a toric face ring $\mathbb{k}[\mathcal{M}]$. This $\mathcal{M}$ comes from a fan, and $\mathbb{k}[\mathcal{M}]$ has a $\mathbb{Z}^{3}$-grading with $\mathbf{M}_{\sigma}=\mathbb{Z}^{3} \cap C_{\sigma}$ for all $\sigma \in \mathcal{X}$. (Find such a grading explicitly.) Next, we assign $\mathbb{k}[x, y, z, w] /(x z-y w)$ to 5 two-dimensional cells and $\mathbb{k}[x, y, z, w, v] /\left(x z-v^{2}, y w-v^{2}\right)$ to the $6^{\text {th }}$ one. Then we get a toric face ring $\mathbb{k}\left[\mathcal{M}^{\prime}\right]$, which is observed in [2, pp. 6-7]. While $\mathbb{k}\left[\mathcal{M}^{\prime}\right]$ admits a $\mathbb{Z}^{3}$-grading and all $\mathbb{k}\left[\mathbf{M}_{\sigma}^{\prime}\right]$ is normal, it is impossible to satisfy $\mathbf{M}_{\sigma}^{\prime}=\mathbb{Z}^{3} \cap C_{\sigma}$ simultaneously for all $\sigma$. A toric face ring without multi-grading is given in Example 2.9.
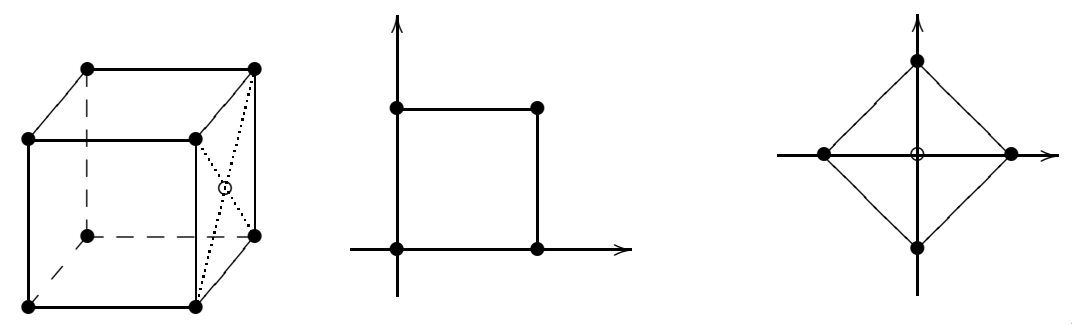

$$
\mathbb{k}[x, y, z, w] /(x z-y w) \quad \mathbb{k}[x, y, z, w, v] /\left(x z-v^{2}, y w-v^{2}\right)
$$

The affine semigroup ring $\mathbb{k}\left[\mathbf{M}_{\sigma}\right]:=\bigoplus_{a \in \mathbf{M}_{\sigma}} \mathbb{k} t^{a}$ can be regarded as a quotient ring of a toric face $\operatorname{ring} R:=\mathbb{k}[\mathcal{M}]$. In the rest of this section, we assume that $\mathbb{k}\left[\mathbf{M}_{\sigma}\right]$ is normal for all $\sigma \in \mathcal{X}$, and set $d:=\operatorname{dim} R=\operatorname{dim} \mathcal{X}+1$.

THEOREM 1.2. In the above situation, the cochain complex $I_{R}^{\bullet}$ given by

$$
I_{R}^{-i}:=\bigoplus_{\substack{\sigma \in \mathcal{X}, \operatorname{dim} \sigma=i-1}} \mathbb{k}\left[\mathbf{M}_{\sigma}\right], \quad I_{R}^{\bullet}: 0 \longrightarrow I_{R}^{-d} \longrightarrow I_{R}^{-d+1} \longrightarrow \cdots \longrightarrow I_{R}^{0} \longrightarrow 0
$$

and

$$
\partial: I_{R}^{-i} \supset \mathbb{k}\left[\mathbf{M}_{\sigma}\right] \ni 1_{\sigma} \longmapsto \sum_{\substack{\operatorname{dim} \mathbb{k}[\tau]=i-1, \tau \subset \bar{\sigma}}} \pm 1_{\tau} \in \bigoplus_{\substack{\operatorname{dim} \mathbb{k}[\tau]=i-1, \tau \subset \bar{\sigma}}} \mathbb{k}\left[\mathbf{M}_{\tau}\right] \subset I_{R}^{-i+1}
$$

is quasi-isomorphic to a normalized dualizing complex $D_{R}^{\bullet}$ of $R$. Here the sign \pm is given by an incidence function of the regular cell complex $\mathcal{X}$.

Clearly, our $I_{R}^{\bullet}$ is analogous to the complex constructed in Ishida [9], but, since we assume that all $\mathbb{k}\left[\mathbf{M}_{\sigma}\right]$ are normal, we do not have to take the 
(graded) injective hull of $\mathbb{k}\left[\mathbf{M}_{\sigma}\right]$. If $\mathcal{M}$ comes from a fan in $\mathbb{R}^{n}$, the above theorem has been obtained in [8, Theorem 5.1] using the $\mathbb{Z}^{n}$-grading of $R$.

We also introduce the notion of $\mathbb{Z} \mathcal{M}$-graded $R$-modules. Since $R$ is not a graded ring, these are not graded modules in the usual sense, but we can consider their "Hilbert functions". In particular, Corollary 6.3, which recaptures a result of [1], gives a formula on the Hilbert function of the local cohomology module $H_{\mathfrak{m}}^{i}(R)$ at the maximal ideal $\mathfrak{m}:=\left(t^{a}|0 \neq a \in| \mathcal{M} \mid\right)$.

In [14], [16], the second author defined squarefree modules $M$ over a normal semigroup ring $\mathbb{k}\left[\mathbf{M}_{\sigma}\right]$, and gave corresponding constructible sheaves $M^{+}$on the closed ball $\bar{\sigma}$. We can extend this to a toric face ring $R$, that is, we define squarefree $R$-modules and associate constructible sheaves on $\mathcal{X}$ with them. In this context, the duality $\mathrm{RHom}_{R}\left(-, I_{R}^{\bullet}\right)$ on the derived category of squarefree $R$-modules corresponds to Poincaré-Verdier duality on the derived category of constructible sheaves on $\mathcal{X}$. For example, the complex $I_{R}^{\bullet}$ consists of squarefree modules, and $\left(I_{R}^{\bullet}\right)^{+}$is the Verdier's dualizing complex of the underlying topological space of $\mathcal{X}$.

COROLlary 1.3. The Buchsbaum property, Cohen-Macaulay property and Gorenstein* property are topological properties of the underlying space of $\mathcal{X}$.

While some parts/cases of Corollary 1.3 have been obtained in existing papers, our argument gives systematic perspective.

\section{$\S 2 . \quad$ Toric face rings}

First, we shall recall the definition of a regular cell complex: A finite regular cell complex (cf. [4, Section 6.2]) is a topological space $X$ together with a finite set $\mathcal{X}$ of subsets of $X$ such that the following conditions are satisfied:

(1) $\emptyset \in \mathcal{X}$ and $X=\bigcup_{\sigma \in \mathcal{X}} \sigma$;

(2) the subsets $\sigma \in \mathcal{X}$ are pairwise disjoint;

(3) for each $\sigma \in \mathcal{X}, \sigma \neq \emptyset$, there exists some $i \in \mathbb{N}$ and a homeomorphism from an $i$-dimensional ball $\left\{x \in \mathbb{R}^{i} \mid\|x\| \leq 1\right\}$ to the closure $\bar{\sigma}$ of $\sigma$ which maps $\left\{x \in \mathbb{R}^{i} \mid\|x\|<1\right\}$ onto $\sigma$.

(4) For any $\sigma \in \mathcal{X}$, the closure $\bar{\sigma}$ can be written as the union of some cells in $\mathcal{X}$. 
An element $\sigma \in \mathcal{X}$ is called a cell. We regard $\mathcal{X}$ as a poset with the order $>$ defined as follows; $\sigma \geq \tau$ if $\bar{\sigma} \supset \tau$. If $\bar{\sigma}$ is homeomorphic to an $i$-dimensional ball, we set $\operatorname{dim} \sigma=i$. Here $\operatorname{dim} \emptyset=-1$. Set $\operatorname{dim} X=$ $\operatorname{dim} \mathcal{X}:=\max \{\operatorname{dim} \sigma \mid \sigma \in \mathcal{X}\}$.

Let $\sigma, \tau \in \mathcal{X}$. If $\operatorname{dim} \sigma=i+1, \operatorname{dim} \tau=i-1$ and $\tau<\sigma$, then there are exactly two cells $\sigma_{1}, \sigma_{2} \in \mathcal{X}$ between $\tau$ and $\sigma$. (Here $\operatorname{dim} \sigma_{1}=\operatorname{dim} \sigma_{2}=i$.) A remarkable property of a regular cell complex is the existence of an incidence function $\varepsilon$ satisfying the following conditions.

(1) To each pair $(\sigma, \tau)$ of cells, $\varepsilon$ assigns a number $\varepsilon(\sigma, \tau) \in\{0, \pm 1\}$.

(2) $\varepsilon(\sigma, \tau) \neq 0$ if and only if $\operatorname{dim} \tau=\operatorname{dim} \sigma-1$ and $\tau<\sigma$.

(3) If $\operatorname{dim} \sigma=i+1, \operatorname{dim} \tau=i-1$ and $\tau<\sigma_{1}, \sigma_{2}<\sigma, \sigma_{1} \neq \sigma_{2}$, then we have

$$
\varepsilon\left(\sigma, \sigma_{1}\right) \varepsilon\left(\sigma_{1}, \tau\right)+\varepsilon\left(\sigma, \sigma_{2}\right) \varepsilon\left(\sigma_{2}, \tau\right)=0 .
$$

We can compute the (co)homology groups of $X$ using the cell decomposition $\mathcal{X}$ and an incidence function $\varepsilon$.

EXAMPLE 2.1. We shall give two typical examples of a finite regular cell complex: one is associated with a simplicial complex $\Delta$ on the vertex set $[n]:=\{1, \ldots, n\}$, i.e., a subset of the power set $2^{[n]}$ such that, for $F, G \in 2^{[n]}$, $F \subset G$ and $G \in \Delta$ imply $F \in \Delta$. Take its geometric realization $\|\Delta\|$, and let $\rho$ be the map giving the realization (see [4] for the definition of a geometric realization). Then $X:=\|\Delta\|$ together with $\{\operatorname{rel}-\operatorname{int}(\rho(F)) \mid F \in \Delta\}$ is a regular cell complex, where rel-int $(\rho(F))$ denotes the relative interior of $\rho(F)$.

The other example is a polytope $P$. In this case, $P$ itself is the underlying topological space; the cells are the relative interiors of its faces.

Definition 2.2. A conical complex consists of the following data.

(1) A finite regular cell complex $\mathcal{X}$ satisfying the intersection property, i.e., for $\sigma, \tau \in \mathcal{X}$, there is a cell $v \in \mathcal{X}$ such that $\bar{v}=\bar{\sigma} \cap \bar{\tau}$;

(2) A set $\Sigma$ of finitely generated cones $C_{\sigma} \subset \mathbb{R}^{\operatorname{dim} \sigma+1}$ with $\sigma \in \mathcal{X}$ and $\operatorname{dim} C_{\sigma}=\operatorname{dim} \sigma+1$.

(3) An injection $\iota_{\sigma, \tau}: C_{\tau} \rightarrow C_{\sigma}$ for $\sigma, \tau \in \mathcal{X}$ with $\sigma \geq \tau$ satisfying the following.

(a) $\iota_{\sigma, \tau}$ can be lifted up to a linear map $\mathbb{R}^{\operatorname{dim} \tau+1} \rightarrow \mathbb{R}^{\operatorname{dim} \sigma+1}$. 
(b) The image $\iota_{\sigma, \tau}\left(C_{\tau}\right)$ is a face of $C_{\sigma}$. Conversely, for a face $C^{\prime}$ of $C_{\sigma}$, there is a sole cell $\tau$ with $\tau \leq \sigma$ such that $\iota_{\sigma, \tau}\left(C_{\tau}\right)=C^{\prime}$. Thus we have a one-to-one correspondence between $\left\{\right.$ faces of $\left.C_{\sigma}\right\}$ and $\{\tau \in \mathcal{X} \mid \tau \leq \sigma\}$.

(c) $\iota_{\sigma, \sigma}=\operatorname{id}_{C_{\sigma}}$ and $\iota_{\sigma, \tau} \circ \iota_{\tau, v}=\iota_{\sigma, v}$ for $\sigma, \tau, v \in \mathcal{X}$ with $\sigma \geq \tau \geq v$.

We denote this structure by $(\Sigma, \mathcal{X})$ or $\Sigma$ simply.

Remark 2.3. (1) We have $\varnothing \in \mathcal{X}$ according to the definition of a regular cell complex, and the corresponding cone $C_{\varnothing}$ is $\{0\}$. Thus for a conical complex $(\Sigma, \mathcal{X})$, each $C_{\sigma} \in \Sigma$ is pointed, i.e., $\{0\}$ is a face of $C_{\sigma}$.

(2) The concept of conical complexes was first defined by Bruns-KochRömer [5] in a slightly different manner, but, under the additional condition that each cone is pointed, their definition is equivalent to ours. That is, our conical complexes are pointed conical complexes of [5].

For grasping the image of a conical complex $(\Sigma, \mathcal{X})$, it is helpful to regard the conical complex as the object given by "gluing" each cones along the injections $\iota_{\sigma, \tau}$. A typical example of a conical complex is a pointed fan, i.e., a finite collection $\Sigma$ of pointed cones in $\mathbb{R}^{n}$ satisfying the following properties:

(1) for $C^{\prime} \subset C \in \Sigma, C^{\prime}$ is a face of $C$ if and only if $C^{\prime} \in \Sigma$;

(2) for $C, C^{\prime} \in \Sigma, C \cap C^{\prime}$ is a common face of $C$ and $C^{\prime}$.

In this case, as an underlying cell complex, we can take $\left\{\operatorname{rel}-i n t\left(C \cap \mathbb{S}^{n-1}\right) \mid\right.$ $C \in \Sigma\}$, where $\mathbb{S}^{n-1}$ denotes the unit sphere in $\mathbb{R}^{n}$, and the injections $\iota$ are inclusion maps.

EXAMPLE 2.4. There exists a conical complex which is not a fan. In fact, consider the Möbius strip as follows.

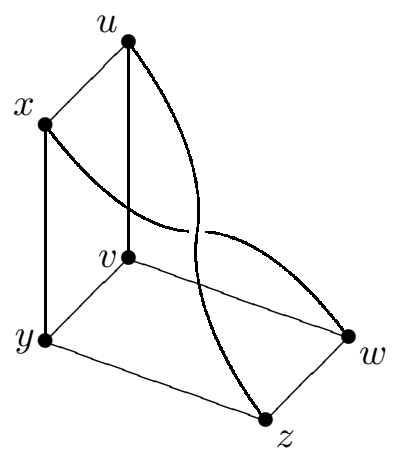


Regarding each rectangles as the cross-sections of 3-dimensional cones, we have a conical complex that is not a fan (see [3]).

A monoidal complex plays a role similar to the defining semigroup of an affine semigroup ring.

Definition 2.5. ([5]) A monoidal complex $\mathcal{M}$ supported by a conical complex $(\Sigma, \mathcal{X})$ is a set of monoids $\left\{\mathbf{M}_{\sigma}\right\}_{\sigma \in \mathcal{X}}$ with the following conditions:

(1) $\mathbf{M}_{\sigma} \subset \mathbb{Z}^{\operatorname{dim} \sigma+1}$ for each $\sigma \in \mathcal{X}$, and it is a finitely generated additive submonoid (so $\mathbf{M}_{\sigma}$ is an affine semigroup);

(2) $\mathbf{M}_{\sigma} \subset C_{\sigma}$ and $\mathbb{R}_{\geq 0} \mathbf{M}_{\sigma}=C_{\sigma}$ for each $\sigma \in \mathcal{X}$ (hence the cone $C_{\sigma}$ is automatically rational);

(3) for $\sigma, \tau \in \mathcal{X}$ with $\sigma \geq \tau$, the map $\iota_{\sigma, \tau}: C_{\tau} \rightarrow C_{\sigma}$ induces an isomorphism $\mathbf{M}_{\tau} \cong \mathbf{M}_{\sigma} \cap \iota_{\sigma, \tau}\left(C_{\tau}\right)$ of monoids.

For example, let $\Sigma$ be a rational pointed fan in $\mathbb{R}^{n}$. Then $\left\{C \cap \mathbb{Z}^{n} \mid C \in\right.$ $\Sigma\}$ gives a monoidal complex. More generally, a family of affine semigroups $\left\{\mathbf{M}_{C} \subset \mathbb{Z}^{n} \mid C \in \Sigma\right\}$ satisfying the following conditions, forms a monoidal complex;

(1) $\mathbb{R}_{\geq 0} \mathbf{M}_{C}=C$ for each $C \in \Sigma$;

(2) $\mathbf{M}_{C} \cap C^{\prime}=\mathbf{M}_{C^{\prime}}$ for $C, C^{\prime} \in \Sigma$ with $C^{\prime} \subset C$.

Remark 2.6. (1) In [2, §2], basic properties of a rational polyhedral complex, which gives a conical complex and a monoidal complex in a natural way, are discussed.

(2) Even if a regular cell complex $\mathcal{X}$ satisfies the intersection property, there does not exist a conical complex of the form $(\Sigma, \mathcal{X})$ in general. For example, there is a simplicial complex $\Delta$ such that the geometric realization $\|\Delta\|$ is homeomorphic to a 3 -dimensional sphere, but $\Delta$ is not the boundary complex of any (4-dimensional) polytope. See, for example, [19, Notes of Chap. 8]. Now take a 4-dimensional ball, and let $\sigma$ be its interior. Triangulating the boundary of the ball, which is a 3-dimensional sphere, according to $\Delta$, we obtain the cell complex $\mathcal{X}:=\Delta \cup\{\sigma\}$ such that $\sigma>\tau$ for all $\tau \in \Delta$. If there is a conical complex of the form $(\Sigma, \mathcal{X})$, then the boundary complex of a cross section of the cone $C_{\sigma} \in \Sigma$ coincides with $\Delta$. This is a contradiction. 
On the other hand, for any 2-dimensional regular cell complex $\mathcal{X}$ satisfying the intersection property, there is a conical complex $(\Sigma, \mathcal{X})$ and a monoidal complex $\mathcal{M}$ supported by it as follows.

Let $n \geq 3$ be an integer. It is an easy exercise to construct an affine semigroup $\mathbf{M}_{n} \subset \mathbb{N}^{3}$ satisfying the following conditions.

(i) The cone $C:=\mathbb{R}_{\geq 0} \mathbf{M}_{n} \subset \mathbb{R}^{3}$ has exactly $n$ extremal rays, that is, its cross section is an $n$-gon.

(ii) For any 2-dimensional face $F$ of $C$, we have $F \cap \mathbf{M}_{n} \cong \mathbb{N}^{2}$ as monoids.

For a 2-dimensional cell $\sigma \in \mathcal{X}$, set $n(\sigma):=\#\{\tau \mid \tau \leq \sigma, \operatorname{dim} \tau=1\}$. By the intersection property of $\mathcal{X}$, we have $n(\sigma) \geq 3$. The assignment $\mathbf{M}_{\sigma}:=\mathbf{M}_{n(\sigma)}$ for each 2-dimensional cell $\sigma$ gives a monoidal complex on $\mathcal{X}$.

For a conical complex $(\Sigma, \mathcal{X})$ and a monoidal complex $\mathcal{M}$ supported by $\Sigma$, we set

$$
|\mathcal{M}|:=\underset{\sigma \in \mathcal{X}}{\lim _{\sigma}} \mathbf{M}_{\sigma}, \quad|\mathbb{Z} \mathcal{M}|:=\underset{\sigma \in \mathcal{X}}{\lim } \mathbb{Z} \mathbf{M}_{\sigma},
$$

where the direct limits are taken with respect to the inclusions $\iota_{\sigma, \tau}: \mathbf{M}_{\tau} \rightarrow$ $\mathbf{M}_{\sigma}$ and induced map $\mathbb{Z} \mathbf{M}_{\tau} \rightarrow \mathbb{Z} \mathbf{M}_{\sigma}$ respectively, for $\sigma, \tau \in \mathcal{X}$ with $\sigma \geq \tau$.

Let $a, b \in|\mathbb{Z} \mathcal{M}|$. If there is some $\sigma \in \mathcal{X}$ with $a, b \in \mathbb{Z} \mathbf{M}_{\sigma}$, by the intersection property of $\mathcal{X}$, there is a unique minimal cell among these $\sigma$ 's. Hence we can define $a \pm b \in|\mathbb{Z} \mathcal{M}|$.

Definition 2.7. ([5]) Let $(\Sigma, \mathcal{X})$ be a conical complex, $\mathcal{M}$ a monoidal complex supported by $\Sigma$, and $\mathbb{k}$ a field. Then the $\mathbb{k}$-vector space

$$
\mathbb{k}[\mathcal{M}]:=\bigoplus_{a \in|\mathcal{M}|} \mathbb{k} t^{a}
$$

where $t$ is a variable, equipped with the following multiplication

$$
t^{a} \cdot t^{b}= \begin{cases}t^{a+b} & \text { if } a, b \in \mathbf{M}_{\sigma} \text { for some } \sigma \in \mathcal{X} \\ 0 & \text { otherwise }\end{cases}
$$

has a $\mathbb{k}$-algebra structure. We call $\mathbb{k}[\mathcal{M}]$ the toric face ring of $\mathcal{M}$ over $\mathbb{k}$.

It is easy to see that $\operatorname{dim} R=\operatorname{dim} \mathcal{X}+1$. When $\Sigma$ is a rational pointed fan, $\mathbb{k}[\mathcal{M}]$ coincides with a toric face ring of Ichim-Römer's sense $([8])$. Moreover, if we choose $C_{\sigma} \cap \mathbb{Z}^{n}$ as $\mathbf{M}_{\sigma}$ for each $\sigma, \mathbb{k}[\mathcal{M}]$ is just an earlier version 
due to Stanley ([12]). Henceforth we refer a toric face ring of $\mathcal{M}$ supported by a fan as an embedded toric face ring. Every Stanley-Reisner ring and every affine semigroup ring (associated with a positive affine semigroup) can be established as embedded toric face rings (see Example 1.1). The most difference between an embedded toric face ring and a non-embedded one, is whether it has a nice $\mathbb{Z}^{n}$-grading or not; an embedded toric face ring always has the natural $\mathbb{Z}^{n}$-grading such that the dimension, as a $\mathbb{k}$-vector space, of each homogeneous component is less than or equal to 1 . However a non-embedded one does not have such a grading.

Toric face rings can be expressed as a quotient ring of a polynomial ring. Let $\mathcal{M}$ be a monoidal complex supported by a conical complex $(\Sigma, \mathcal{X})$, and $\left\{a_{e}\right\}_{e \in E}$ a family of elements of $|\mathcal{M}|$ generating $\mathbb{k}[\mathcal{M}]$ as a $\mathbb{k}$-algebra, or equivalently, $\left\{a_{e}\right\}_{e \in E} \cap \mathbf{M}_{\sigma}$ generates $\mathbf{M}_{\sigma}$ for each $\sigma \in \mathcal{X}$. Then the polynomial ring $S:=\mathbb{k}\left[X_{e} \mid e \in E\right]$ surjects on $\mathbb{k}[\mathcal{M}]$. We denote, by $I_{\mathcal{M}}$, its kernel. Similarly we have the surjection $S_{\sigma}:=\mathbb{k}\left[X_{e} \mid a_{e} \in \mathcal{M}_{\sigma}\right.$, e $\in$ $E] \rightarrow \mathbb{k}\left[\mathbf{M}_{\sigma}\right]$, where $\mathbb{k}\left[\mathbf{M}_{\sigma}\right]$ denotes the affine semigroup ring of $\mathbf{M}_{\sigma}$, and denote its kernel by $I_{\mathbf{M}_{\sigma}}$.

Proposition 2.8. ([5, Proposition 2.6]) With the above notation, we have

$$
I_{\mathcal{M}}=A_{\mathcal{M}}+\sum_{i=1}^{n} S I_{\mathbf{M}_{\sigma_{i}}}
$$

where $\sigma_{1}, \ldots, \sigma_{n}$ are the maximal cells of $\mathcal{X}$, and $A_{\mathcal{M}}$ is the ideal of $S$ generated by the squarefree monomials $\prod_{h \in H} X_{h}$ for which $\left\{a_{h} \mid h \in H\right\}$ is not contained in $\mathbf{M}_{\sigma}$ for any $\sigma \in \mathcal{X}$.

Example 2.9. ([5, Example 4.6]) Consider the conical complex given in Example 2.4, and choose each rectangles to be a unit square. In this case, we can construct a monoidal complex $\mathcal{M}$ such that $\mathbf{M}_{\sigma}=C_{\sigma} \cap \mathbb{Z}^{\operatorname{dim} C_{\sigma}}$ for all $\sigma$, and then $u, v, w, x, y, z$ are generators of $\mathcal{M}$. We set $S:=$ $\mathbb{k}\left[X_{u}, X_{v}, X_{w}, X_{x}, X_{y}, X_{z}\right]$, where $X_{u}, \ldots, X_{z}$ are variables. Clearly, $\mathbb{k}\left[\mathbf{M}_{\sigma}\right]$ is a polynomial ring if $\operatorname{dim} \sigma \leq 1$, and one of the following

$$
\begin{aligned}
& \mathbb{k}\left[X_{u}, X_{v}, X_{x}, X_{y}\right] /\left(X_{x} X_{v}-X_{u} X_{y}\right), \\
& \mathbb{k}\left[X_{v}, X_{w}, X_{y}, X_{z}\right] /\left(X_{v} X_{z}-X_{y} X_{w}\right), \\
& \mathbb{k}\left[X_{u}, X_{w}, X_{x}, X_{z}\right] /\left(X_{x} X_{z}-X_{u} X_{w}\right),
\end{aligned}
$$


if $\operatorname{dim} \sigma=2$. Therefore we conclude that

$$
\begin{array}{r}
I_{\mathcal{M}}=\left(X_{x} X_{v}-X_{u} X_{y}, X_{v} X_{z}-X_{y} X_{w}, X_{x} X_{z}-X_{u} X_{w}\right. \\
\left.\quad X_{u} X_{v} X_{w}, X_{u} X_{v} X_{z}\right) \subset S .
\end{array}
$$

We leave the reader to verify that the other squarefree monomials in $A_{\mathcal{M}}$, e.g. $X_{x} X_{y} X_{z}$, are indeed contained in the above ideal.

In this paper, we often assume that $\mathbb{k}[\mathcal{M}]$ satisfies the following condition.

DEFINITION 2.10. We say a toric face ring $\mathbb{k}[\mathcal{M}]$ (or a monoidal complex $\mathcal{M})$ is cone-wise normal, if the affine semigroup ring $\mathbb{k}\left[\mathbf{M}_{\sigma}\right]$ is normal for all $\sigma \in \mathcal{X}$.

If $\mathbb{k}[\mathcal{M}]$ is cone-wise normal, then $\mathbb{k}\left[\mathbf{M}_{\sigma}\right]$ is Cohen-Macaulay for all $\sigma \in \mathcal{X}$. Clearly, the toric face rings given in Examples 1.1 and 2.9 are cone-wise normal.

Remark 2.11. The notion of a cone-wise normal monoidal complex $\mathcal{M}$ is equivalent to that of the lattice points $\mathcal{W} F\left(\Pi_{\text {rat }}\right)$ of a weak fan $\mathcal{W} F$ introduced by Bruns and Gubeladze in [2, Definition 2.6]. In this case, our ring $\mathbb{k}[\mathcal{M}]$ is the same thing as the ring $\mathbb{k}[\mathcal{W} F]$ of $[2]$.

An affine semigroup ring $A=\mathbb{k}\left[\mathbf{M}_{\sigma}\right]$ has a graded ring structure $A=$ $\bigoplus_{i \in \mathbb{N}} A_{i}$ with $A_{0}=\mathbb{k}$. The toric face ring given in Example 2.9 also has an $\mathbb{N}$-grading given by $\operatorname{deg} X_{u}=\cdots=\operatorname{deg} X_{z}=1$. This is not true in general; there is a monoidal complex whose toric face ring does not have an $\mathbb{N}$-grading. See [2, Example 2.7].

For a commutative $\operatorname{ring} A$, let $\operatorname{Mod} A(\operatorname{resp} . \bmod A)$ denote the category of (resp. finitely generated) $A$-modules.

Definition 2.12. Let $R:=\mathbb{k}[\mathcal{M}]$ be a toric face ring of a monoidal complex $\mathcal{M}$ supported by a conical complex $(\Sigma, \mathcal{X})$.

(1) $M \in \operatorname{Mod} R$ is said to be $\mathbb{Z} \mathcal{M}$-graded if the following conditions are satisfied;

(a) $M=\bigoplus_{a \in|\mathbb{Z} \mathcal{M}|} M_{a}$ as $\mathbb{k}$-vector spaces; 
(b) $t^{a} \cdot M_{b} \subset M_{a+b}$ if $a \in \mathbf{M}_{\sigma}$ and $b \in \mathbb{Z} \mathbf{M}_{\sigma}$ for some $\sigma \in \mathcal{X}$, and $t^{a} \cdot M_{b}=0$ otherwise.

(2) $M \in \operatorname{Mod} R$ is said to be $\mathcal{M}$-graded if it is $\mathbb{Z} \mathcal{M}$-graded and $M_{a}=0$ for $a \notin|\mathcal{M}|$.

Of course, setting $R_{a}:=\mathbb{k} t^{a}$ for each $a \in|\mathcal{M}|$, we see that $R$ itself is $|\mathcal{M}|$-graded. Any monomial ideal, i.e., an ideal generated by elements of the form $t^{a}$ for some $a \in|\mathcal{M}|$, is $\mathcal{M}$-graded, and hence $\mathbb{Z} \mathcal{M}$-graded. Conversely, every $\mathbb{Z} \mathcal{M}$-graded ideal is a monomial ideal.

Let $\operatorname{Mod}_{\mathbb{Z} \mathcal{M}} R\left(\right.$ resp. $\bmod _{\mathbb{Z} \mathcal{M}} R$ ) denote the subcategory of Mod $R$ (resp. $\bmod R$ ) whose objects are $\mathbb{Z} \mathcal{M}$-graded $R$-modules and morphisms are degree preserving maps, i.e., $R$-homomorphisms $f: M \rightarrow N$ such that $f\left(M_{a}\right) \subset N_{a}$ for $a \in|\mathbb{Z} \mathcal{M}|$. It is clear that $\operatorname{Mod}_{\mathbb{Z} \mathcal{M}} R$ and $\bmod _{\mathbb{Z} \mathcal{M}} R$ are abelian.

For each $\sigma \in \mathcal{X}$, the ideal $\mathfrak{p}_{\sigma}:=\left(t^{a} \mid a \notin \mathbf{M}_{\sigma}\right) \subset R$ is a $\mathbb{Z} \mathcal{M}$-graded prime ideal since $R / \mathfrak{p}_{\sigma} \cong \mathbb{k}\left[\mathbf{M}_{\sigma}\right]$. Conversely, every $\mathbb{Z} \mathcal{M}$-graded prime ideals are of this form.

Lemma 2.13. There is a one-to-one correspondence between the cells in $\mathcal{X}$ and the $\mathbb{Z} \mathcal{M}$-graded prime ideals of $R$.

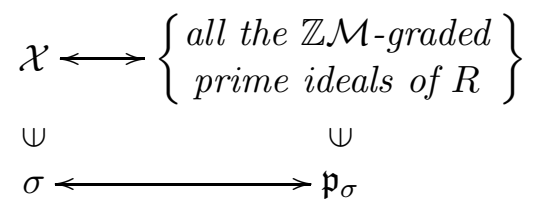

Proof. The proof is quite the same as [8, Lemma 2.1].

For an ideal $I$ of $R$, we denote, by $I^{*}$, the ideal of $R$ generated by all the monomials belonging to $I$. As in the case of a usual grading, we have the following:

LEMma 2.14. For a prime ideal $\mathfrak{p}$ of $R, \mathfrak{p}^{*}$ is also prime, and hence is a $\mathbb{Z} \mathcal{M}$-graded prime ideal.

Proof. Since the ideal 0 can be decomposed as follows

$$
\bigcap_{\substack{\sigma \in \mathcal{X} \\ \sigma: \text { maximal }}} \mathfrak{p}_{\sigma}=0,
$$


$\left\{\mathfrak{p}_{\sigma} \mid \sigma\right.$ is a maximal cell of $\left.\mathcal{X}\right\}$ is the set of minimal primes of $R$. Hence $\mathfrak{p}$ must contain $\mathfrak{p}_{\sigma}$ for some $\sigma \in \mathcal{X}$. It follows that $\mathfrak{p}^{*} \supset \mathfrak{p}_{\sigma}$. Consider the images $\rho(\mathfrak{p})$ and $\rho\left(\mathfrak{p}^{*}\right)$ by the surjection $\rho: R \rightarrow \mathbb{k}\left[\mathbf{M}_{\sigma}\right]$. Then $\rho(\mathfrak{p})$ is prime and $\rho\left(\mathfrak{p}^{*}\right)$ is the ideal generated by the monomials contained in $\rho(\mathfrak{p})$, whence is prime. Therefore we conclude that $\mathfrak{p}^{*}$ is also prime.

Corollary 2.15. Let $\mathfrak{a}$ be a $\mathbb{Z} \mathcal{M}$-graded ideal of $R$. Then its radical ideal $\sqrt{\mathfrak{a}}$ is also $\mathbb{Z} \mathcal{M}$-graded.

Proof. Since $\mathfrak{a} \subset \mathfrak{p}^{*}$ holds for a prime ideal $\mathfrak{p}$ with $\mathfrak{a} \subset \mathfrak{p}$, we have

$$
\bigcap_{\mathfrak{p} \supset \mathfrak{a}} \mathfrak{p}^{*} \subset \bigcap_{\mathfrak{p} \supset \mathfrak{a}} \mathfrak{p}=\sqrt{\mathfrak{a}} \subset \bigcap_{\mathfrak{p} \supset \mathfrak{a}} \mathfrak{p}^{*},
$$

and therefore $\sqrt{\mathfrak{a}}=\bigcap_{\mathfrak{p} \supset \mathfrak{a}} \mathfrak{p}^{*}$.

\section{§3. Cěch complexes and local cohomologies}

Let $(\Sigma, \mathcal{X})$ be a conical complex, and $\mathcal{M}$ a monoidal complex. For $\sigma \in$ $\mathcal{X}$, set $T_{\sigma}:=\left\{t^{a} \mid a \in \mathbf{M}_{\sigma}\right\} \subset R:=\mathbb{k}[\mathcal{M}]$. Then $T_{\sigma}$ forms a multiplicatively closed subset consisting of monomials. Moreover, a multiplicatively closet subset $T$ consisting of monomials is contained in some $T_{\sigma}$, unless $T \ni 0$.

Lemma 3.1. Let $M \in \operatorname{Mod}_{\mathbb{Z M}} R$, and let $T$ be a multiplicatively closed subset of $R$ consisting of monomials. Then $T^{-1} M \in \operatorname{Mod}_{\mathbb{Z} \mathcal{M}} R$.

Proof. Take any $x / t^{a} \in T^{-1} M$ with $a \in|\mathcal{M}|, b \in|\mathbb{Z} \mathcal{M}|$, and $x \in M_{b}$. If there is no $\sigma \in \mathcal{X}$ with $a, b \in \mathbb{Z} \mathbf{M}_{\sigma}$, then $x / t^{a}=\left(x t^{a}\right) / t^{2 a}=0$; otherwise, $b-a$ is well-defined and in $|\mathbb{Z} \mathcal{M}|$. Now for $\lambda \in|\mathbb{Z} \mathcal{M}|$, set

$$
\left(T^{-1} M\right)_{\lambda}:=\sum_{x \in M_{b}, b-a=\lambda} \mathbb{k} \cdot \frac{x}{t^{a}}
$$

Then we have $T^{-1} M=\bigoplus_{\lambda \in|\mathbb{Z M}|}\left(T^{-1} M\right)_{\lambda}$ as $\mathbb{k}$-vector spaces, which gives $T^{-1} M$ a $|\mathbb{Z} \mathcal{M}|$-grading.

Well, set

$$
L_{R}^{i}:=\bigoplus_{\substack{\sigma \in \mathcal{X} \\ \operatorname{dim} \sigma=i-1}} T_{\sigma}^{-1} R
$$


and define $\partial: L_{R}^{i} \rightarrow L_{R}^{i+1}$ by

$$
\partial(x)=\sum_{\substack{\tau \geq \sigma \\ \operatorname{dim} \tau=i}} \varepsilon(\tau, \sigma) \cdot f_{\tau, \sigma}(x)
$$

for $x \in T_{\sigma}^{-1} R \subset L_{R}^{i}$, where $\varepsilon$ is an incidence function on $\mathcal{X}$ and $f_{\tau, \sigma}$ is the natural map $T_{\sigma}^{-1} R \rightarrow T_{\tau}^{-1} R$ for $\sigma \leq \tau$. Then $\left(L_{R}^{\bullet}, \partial\right)$ forms a complex in $\operatorname{Mod}_{\mathbb{Z} \mathcal{M}} R$ :

$$
L_{R}^{\bullet}: 0 \longrightarrow L_{R}^{0} \stackrel{\partial}{\longrightarrow} L_{R}^{1} \stackrel{\partial}{\longrightarrow} \cdots \stackrel{\partial}{\longrightarrow} L_{R}^{d} \longrightarrow 0,
$$

where $d=\operatorname{dim} R=\operatorname{dim} \mathcal{X}+1$. We set $\mathfrak{m}:=\left(t^{a}|0 \neq a \in| \mathcal{M} \mid\right)$. This is a maximal ideal of $R$.

Proposition 3.2. (cf. [8, Theorem 4.2]) For any $R$-module $M$,

$$
H_{\mathfrak{m}}^{i}(M) \cong H^{i}\left(L_{R}^{\bullet} \otimes_{R} M\right)
$$

for all $i$.

Proof. It suffices to show the following:

(1) $H^{0}\left(L_{R}^{\bullet} \otimes_{R} M\right) \cong H_{\mathfrak{m}}^{0}(M)$;

(2) for a short exact sequence $0 \rightarrow M_{1} \rightarrow M_{2} \rightarrow M_{3} \rightarrow 0$ in $\operatorname{Mod} R$, the induced one $0 \rightarrow L_{R}^{\bullet} \otimes_{R} M_{1} \rightarrow L_{R}^{\bullet} \otimes_{R} M_{2} \rightarrow L_{R}^{\bullet} \otimes_{R} M_{3} \rightarrow 0$ is also exact;

(3) for any injective $R$-module $I, H^{i}\left(L_{R}^{\bullet} \otimes_{R} I\right)=0$ for all $i \geq 1$.

Let $\mathfrak{a}$ be the ideal generated by elements $t^{a}$ with $0 \neq a \in C_{\sigma}$ for some 1-dimensional cone $C_{\sigma}$. Since $\operatorname{Ker}\left(L_{R}^{0} \otimes_{R} M \rightarrow L_{R}^{1} \otimes_{R} M\right)=H_{\mathfrak{a}}^{0}(M)$, to prove (1), we only have to show that $\sqrt{\mathfrak{a}}=\mathfrak{m}$. Let $\mathfrak{p}$ be a prime containing $\mathfrak{a}$. Since $\mathfrak{a}$ is graded, we have $\mathfrak{p}^{*} \supset \mathfrak{a}$. Thus there exists $\tau \in \mathcal{X}$ such that $\mathfrak{p}_{\tau} \supset \mathfrak{a}$, but then $C_{\tau}$ contains no 1-dimensional face. Therefore we conclude that $\mathfrak{p}_{\tau}=\mathfrak{p}_{\varnothing}=\mathfrak{m}$, which implies $\sqrt{\mathfrak{a}}=\mathfrak{m}$.

The condition (2) follows easily from the flatness of the localization. For (3), we can apply the same argument of Ichim and Römer [8] for embedded toric face rings (but we need to use Lemma 2.14).

Let $\mathrm{R} \Gamma_{\mathfrak{m}}: D^{b}(\operatorname{Mod} R) \rightarrow D^{b}(\operatorname{Mod} R)$ be the right derived functor of $\Gamma_{\mathfrak{m}}:=\lim _{\longrightarrow} \operatorname{Hom}\left(R / \mathfrak{m}^{n},-\right)$, where $D^{b}(\operatorname{Mod} R)$ is the bounded derived category of $\operatorname{Mod} R$. Recall that $H^{i}\left(\mathrm{R} \Gamma_{\mathfrak{m}}(M)\right)=H_{\mathfrak{m}}^{i}(M)$ for all $i$ and 
$M \in \operatorname{Mod} R$. The usual spectral sequence argument of double complexes tells us that $L_{R}^{\bullet}$ is a flat resolution of $\mathrm{R}_{\mathfrak{m}}(R)$, and therefore we have the following.

Corollary 3.3. For a bounded complex $M^{\bullet}$ of $R$-modules, $\mathrm{R} \Gamma_{\mathfrak{m}}\left(M^{\bullet}\right)$ and $L_{R}^{\bullet} \otimes_{R} M^{\bullet}$ are isomorphic in $D^{b}(\operatorname{Mod} R)$.

When $M$ is $\mathbb{Z} \mathcal{M}$-graded, by Lemma $3.1, T_{\sigma}^{-1} R \otimes_{R} M$ is also $\mathbb{Z} \mathcal{M}$ graded, and moreover the differentials of $L_{R}^{\bullet} \otimes_{R} M$ are in $\operatorname{Mod}_{\mathbb{Z} \mathcal{M}} R$. Thus if $M \in \operatorname{Mod}_{\mathbb{Z} \mathcal{M}} R, H^{i}\left(L_{R}^{\bullet} \otimes_{R} M\right)$ has a $\mathbb{Z} \mathcal{M}$-grading induced by $L_{R}^{\bullet} \otimes M$. Hence we have the following.

Corollary 3.4. $H_{\mathfrak{m}}^{i}(M) \in \operatorname{Mod}_{\mathbb{Z} \mathcal{M}} R$ for $M \in \operatorname{Mod}_{\mathbb{Z M}} R$.

\section{§4. Squarefree Modules}

In this section, we assume that all the toric face rings are cone-wise normal. Let $(\Sigma, \mathcal{X})$ be a conical complex, $\mathcal{M}$ a monoidal complex, and $R$ the toric face ring of $\mathcal{M}$. For $a \in|\mathcal{M}|$, there exists a unique cell $\sigma \in \mathcal{X}$ such that rel-int $\left(C_{\sigma}\right) \ni a$. We denote this $\sigma$ by $\operatorname{supp}(a)$.

Definition 4.1. An $R$-module $M \in \bmod _{\mathbb{Z} \mathcal{M}} R$ is said to be squarefree if it is $\mathcal{M}$-graded and the multiplication map $M_{a} \ni x \mapsto t^{b} x \in M_{a+b}$ is an isomorphism of $\mathbb{k}$-vector spaces for all $a, b \in|\mathcal{M}|$ with $\operatorname{supp}(a+b)=\operatorname{supp}(a)$.

For a monomial ideal $I$ of $R$, it is a squarefree $R$-module, if and only if so is $R / I$, if and only if $I=\sqrt{I}$. In particular, $\mathfrak{p}_{\sigma}$ and $R / \mathfrak{p}_{\sigma}$ are squarefree. We denote, by $\mathrm{Sq} R$, the full subcategory of $\bmod _{\mathbb{Z} \mathcal{M}} R$ consisting of squarefree $R$-modules. As in the case of affine semigroup rings or Stanley-Reisner rings (see [14], [15]), Sq $R$ has nice properties. Since their proofs are also quite similar to these cases, we omit some of them.

Lemma 4.2. (cf. [14], [15]) Let $M \in \mathrm{Sq} R$. Then for $a, b \in|\mathcal{M}|$ with $\operatorname{supp}(a) \geq \operatorname{supp}(b)$, there exists a $\mathbb{k}$-linear map $\varphi_{a, b}^{M}: M_{b} \rightarrow M_{a}$ satisfying the following properties:

(1) $\varphi_{a, b}^{M}$ is bijective if $\operatorname{supp}(a)=\operatorname{supp}(b)$;

(2) $\varphi_{a, a}^{M}=\mathrm{id}$ and $\varphi_{a, b}^{M} \circ \varphi_{b, c}^{M}=\varphi_{a, c}^{M}$ for $a, b, c \in|\mathcal{M}|$ with $\operatorname{supp}(c) \leq$ $\operatorname{supp}(b) \leq \operatorname{supp}(a)$ 
(3) For $a, a^{\prime}, b, b^{\prime} \in|\mathcal{M}|$ with $\operatorname{supp}(a) \leq \operatorname{supp}\left(a^{\prime}\right)$ and $\operatorname{supp}(a+b) \leq$ $\operatorname{supp}\left(a^{\prime}+b^{\prime}\right)$, the following diagram

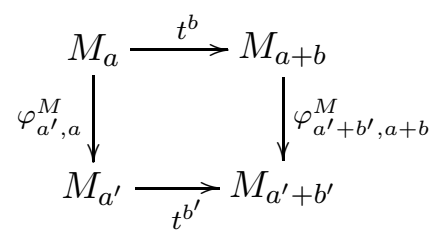

commutes.

Let $\Lambda$ denote the incidence algebra of the regular cell complex $\mathcal{X}$ over $\mathbb{k}$ (regarding $\mathcal{X}$ as a poset by its order $>$ ). That is, $\Lambda$ is a finite dimensional associative $\mathbb{k}$-algebra with basis $\left\{e_{\sigma, \tau} \mid \sigma, \tau \in \mathcal{X}\right.$ with $\left.\sigma \geq \tau\right\}$, and its multiplication is defined by

$$
e_{\sigma, \tau} \cdot e_{\tau^{\prime}, v}= \begin{cases}e_{\sigma, v} & \text { if } \tau=\tau^{\prime} \\ 0 & \text { otherwise }\end{cases}
$$

We write $e_{\sigma}:=e_{\sigma, \sigma}$ for $\sigma \in \mathcal{X}$. Each $e_{\sigma}$ is idempotent, and moreover $\Lambda e_{\sigma}$ is indecomposable as a left $\Lambda$-module. It is easy to verify that $e_{\sigma} \cdot e_{\tau}=0$ if $\sigma \neq \tau$ and that $1=\sum_{\sigma \in \mathcal{X}} e_{\sigma}$. Hence $\Lambda$, as a left $\Lambda$-module, can be decomposed as $\Lambda=\bigoplus_{\sigma \in \mathcal{X}} \Lambda e_{\sigma}$.

Let $\bmod \Lambda$ denote the category of finitely generated left $\Lambda$-modules. As a $\mathbb{k}$-vector space, any $M \in \bmod \Lambda$ has the decomposition $M=\bigoplus_{\sigma \in \mathcal{X}} e_{\sigma} M$. Henceforth we set $M_{\sigma}:=e_{\sigma} M$.

For each $\sigma \in \mathcal{X}$, we can construct an indecomposable injective object in $\bmod \Lambda$ as follows; set

$$
\bar{E}(\sigma):=\bigoplus_{\tau \in \mathcal{X}, \tau \leq \sigma} \mathbb{k} \bar{e}_{\tau}
$$

where $\bar{e}_{\tau}$ 's are basis elements. The multiplication on $\bar{E}(\sigma)$ from the left defined by

$$
e_{v, \omega} \cdot \bar{e}_{\tau}= \begin{cases}\bar{e}_{v} & \text { if } \tau=\omega \text { and } v \leq \sigma \\ 0 & \text { otherwise }\end{cases}
$$

bring $\bar{E}(\sigma)$ a left $\Lambda$-module structure. The following is well known.

Proposition 4.3. The category $\bmod \Lambda$ is abelian and enough injectives, and any indecomposable injective object is isomorphic to $\bar{E}(\sigma)$ for some $\sigma \in \mathcal{X}$. 
As in the case of affine semigroup rings and Stanley-Reisner rings, we have

Proposition 4.4. (cf. [14], [15]) There is an equivalence between $\mathrm{Sq} R$ and $\bmod \Lambda$. Hence $\mathrm{Sq} R$ is abelian, and enough injectives. Any indecomposable injective object in $\mathrm{Sq} R$ is isomorphic to $R / \mathfrak{p}_{\sigma}$ for some $\sigma \in \mathcal{X}$.

Proof. First, we will show the category equivalence. The object $M \in$ Sq $R$ corresponding to $N \in \bmod \Lambda$ is given as follows. Set $M_{a}:=N_{\operatorname{supp}(a)}$ for each $a \in|\mathcal{M}|$. For $a, b \in|\mathcal{M}|$ such that $a+b$ exists, define the multiplication $M_{a} \ni x \mapsto t^{b} \cdot x \in M_{a+b}$ by

$$
M_{a}=N_{\operatorname{supp}(a)} \ni x \longmapsto e_{\operatorname{supp}(a+b), \operatorname{supp}(a)} \cdot x \in N_{\operatorname{supp}(a+b)}=M_{a+b} .
$$

Then $M$ becomes a squarefree module. See [14], [15] for details (though right $\Lambda$-modules are treated in [14], [15], there is no essential difference).

Since $R / \mathfrak{p}_{\sigma}$ corresponds to $\bar{E}(\sigma)$ in this equivalence, the other statements follow from Proposition 4.3.

Let $D^{b}(\mathrm{Sq} R)$ be the bounded derived category of Sq $R$. We shall define the functor $\mathbb{D}: D^{b}(\mathrm{Sq} R) \rightarrow D^{b}(\mathrm{Sq} R)^{\mathrm{op}}$. This functor will play an important role in the next section. First, we choose elements $a(\sigma) \in|\mathcal{M}|$ with $\operatorname{supp}(a(\sigma))=\sigma$ for each $\sigma \in \mathcal{X}$, and set $\varphi_{\sigma, \tau}^{M}:=\varphi_{a(\sigma), a(\tau)}^{M}$ for $M \in \operatorname{Sq} R$ and $\sigma, \tau \in \mathcal{X}$ with $\tau \leq \sigma$, where $\varphi_{a(\sigma), a(\tau)}^{M}$ is the map given in Lemma 4.2. To a bounded complex $M^{\bullet}$ of squarefree $R$-modules, we assign the complex $\mathbb{D}\left(M^{\bullet}\right)$ defined as follows: the component of cohomological degree $p$ is

$$
\mathbb{D}\left(M^{\bullet}\right)^{p}:=\bigoplus_{i+\operatorname{dim} C_{\sigma}=-p}\left(M_{a(\sigma)}^{i}\right)^{*} \otimes_{\mathbb{k}} R / \mathfrak{p}_{\sigma},
$$

where $(-)^{*}$ denotes the $\mathbb{k}$-dual, but the "degree" of $\left(M_{a(\sigma)}^{i}\right)^{*}$ is $0 \in|\mathbb{Z} \mathcal{M}|$. Define $d^{\prime}: \mathbb{D}\left(M^{\bullet}\right)^{p} \rightarrow \mathbb{D}\left(M^{\bullet}\right)^{p+1}$ and $d^{\prime \prime}: \mathbb{D}\left(M^{\bullet}\right)^{p} \rightarrow \mathbb{D}\left(M^{\bullet}\right)^{p+1}$ by

$$
\begin{aligned}
d^{\prime}(y \otimes r) & =\sum_{\substack{\tau \leq \sigma, \operatorname{dim} \tau=\operatorname{dim} \sigma-1}} \varepsilon(\sigma, \tau) \cdot\left(\varphi_{\sigma, \tau}^{M^{i}}\right)^{*}(y) \otimes g_{\tau, \sigma}(r), \\
d^{\prime \prime}(y \otimes r)= & (-1)^{p} \cdot\left(\partial_{M}^{i} \bullet\right)^{*}(y) \otimes r
\end{aligned}
$$

for $y \in M_{a(\sigma)}^{i}$ with $i+\operatorname{dim} C_{\sigma}=-p$ and $r \in R / \mathfrak{p}_{\sigma}$. Here $\varepsilon(\sigma, \tau)$ is an incidence function on $\mathcal{X}$ and $g_{\tau, \sigma}: R / \mathfrak{p}_{\sigma} \rightarrow R / \mathfrak{p}_{\tau}$ is the surjection induced 
by the inclusion $\mathfrak{p}_{\sigma} \subset \mathfrak{p}_{\tau}$. Clearly, $\left(\mathbb{D}\left(M^{\bullet}\right), d^{\prime}+d^{\prime \prime}\right)$ forms a bounded complex in $\mathrm{Sq} R$, and Lemma 4.2 guarantees the independence of $\mathbb{D}\left(M^{\bullet}\right)$ from the choice of $a(\sigma)$ 's.

Let $K^{b}(\mathrm{Sq} R)$ be the bounded homotopy category of $\mathrm{Sq} R$. Since the above assignment preserves mapping cones, it gives a triangulated functor of $K^{b}(\mathrm{Sq} R) \rightarrow K^{b}(\mathrm{Sq} R)^{\mathrm{op}}$, and an usual argument using spectral sequences indicates that it preserves quasi-isomorphisms. Hence it induces the functor $D^{b}(\mathrm{Sq} R) \rightarrow D^{b}(\mathrm{Sq} R)^{\text {op }}$, which is denoted by $\mathbb{D}$ again.

Up to translation, the functor $\mathbb{D}$ coincides with the functor $\mathbf{D}$ : $D^{b}(\bmod \Lambda) \rightarrow D^{b}(\bmod \Lambda)^{\text {op }}$ defined in [17], through the equivalence Sq $R \cong$ $\bmod \Lambda$ in Proposition 4.4. Hence by $[17$, Theorem $3.4(1)]$, we have the following.

Proposition 4.5. The functor $\mathbb{D}: D^{b}(\mathrm{Sq} R) \rightarrow D^{b}(\mathrm{Sq})^{\text {op }}$ satisfies $\mathbb{D} \circ$ $\mathbb{D} \cong \mathrm{id}$.

\section{§5. Dualizing complexes}

We first recall the following useful result due to Sharp ([11]).

TheOREM 5.1. (Sharp) Let $A$ and $B$ be commutative noetherian rings, and $f: A \rightarrow B$ a ring homomorphism. Assume that $A$ has a dualizing complex $D_{A}^{\bullet}$ and $B$, regarded as an $A$-module by $f$, is finitely generated. Then $\operatorname{Hom}_{A}\left(B, D_{A}^{\bullet}\right)$ is a dualizing complex of $B$.

For a commutative ring $A$, we denote, by $E_{A}(-)$, the injective hull in $\operatorname{Mod} A$. Let $(\Sigma, \mathcal{X})$ be a conical complex, $\mathcal{M}$ a cone-wise normal monoidal complex supported by $\Sigma$, and $R:=\mathbb{k}[\mathcal{M}]$ its toric face ring. Since $R$ is a finitely generated $\mathbb{k}$-algebra, we can take a polynomial ring which surjects onto $R$. Thus, Proposition 5.1 implies that $R$ has a normalized dualizing complex

$$
\begin{gathered}
D_{R}^{\bullet}: 0 \longrightarrow \bigoplus_{\substack{\mathfrak{p} \in \operatorname{Spec} R, \operatorname{dim} R / \mathfrak{p}=d}} E_{R}(R / \mathfrak{p}) \longrightarrow \bigoplus_{\substack{\mathfrak{p} \in \operatorname{Spec} R, \operatorname{dim} R / \mathfrak{p}=d-1}} E_{R}(R / \mathfrak{p}) \longrightarrow \cdots \\
\cdots \longrightarrow \bigoplus_{\substack{\mathfrak{p} \in \operatorname{Spec} R, \operatorname{dim} R / \mathfrak{p}=0}}^{\longrightarrow} E_{R}(R / \mathfrak{p}) \longrightarrow 0
\end{gathered}
$$


where $d:=\operatorname{dim} R=\operatorname{dim} \mathcal{X}+1$ and cohomological degrees are given by

$$
D_{R}^{i}:=\bigoplus_{\substack{\mathfrak{p} \in \operatorname{Spec} R, \operatorname{dim} R / \mathfrak{p}=-i}} E_{R}(R / \mathfrak{p}) .
$$

On the other hand, set

$$
I_{R}^{i}:=\bigoplus_{\substack{\sigma \in \mathcal{X} \\ \operatorname{dim} R / \mathfrak{p}_{\sigma}=-i}} R / \mathfrak{p}_{\sigma}
$$

for $i=0, \ldots, d$, and define $I_{R}^{-i} \rightarrow I_{R}^{-i+1}$ by

$$
x \longmapsto \sum_{\substack{\operatorname{dim} \mathbb{k}[\tau]=i-1 \\ \tau \leq \sigma}} \varepsilon(\sigma, \tau) \cdot g_{\tau, \sigma}(x)
$$

for $x \in R / \mathfrak{p}_{\sigma} \subset I_{R}^{-i}$, where $\varepsilon(\sigma, \tau)$ denotes an incidence function of $\mathcal{X}$, and $g_{\tau, \sigma}$ is the surjection $R / \mathfrak{p}_{\sigma} \rightarrow R / \mathfrak{p}_{\tau}$. Then

$$
I_{R}^{\bullet}: 0 \longrightarrow I_{R}^{-d} \longrightarrow I_{R}^{-d+1} \longrightarrow \cdots \longrightarrow I_{R}^{0} \longrightarrow 0
$$

is a complex.

THEOREM 5.2. With the above situation (in particular, $R$ is cone-wise normal), $I_{R}^{\bullet}$ is quasi-isomorphic to the normalized dualizing complex $D_{R}^{\bullet}$ of $R$.

For the embedded case, Theorem 5.2 was already shown by Ichim and Römer [8], using the natural $\mathbb{Z}^{n}$-graded structure. However, in the general case, we cannot apply the same argument.

Proposition 5.3. With the hypothesis in Theorem 5.2, $I_{R}^{\bullet}$ is a subcomplex of $D_{R}^{\bullet}$.

Proof. We shall go through some steps.

Step 1. Some observations.

For $\sigma \in \mathcal{X}$, we set $\mathbb{k}[\sigma]:=R / \mathfrak{p}_{\sigma} \cong \mathbb{k}\left[\mathbf{M}_{\sigma}\right]$ and $d_{\sigma}:=\operatorname{dim} C_{\sigma}=$ $\operatorname{dim} \mathbb{k}[\sigma]=\operatorname{dim} \sigma+1$. Note that

$$
D_{\sigma}^{\bullet}:=\operatorname{Hom}_{R}\left(\mathbb{k}[\sigma], D_{R}^{\bullet}\right)
$$


is a normalized dualizing complex of $\mathbb{k}[\sigma]$ by Proposition 5.1. Since $\mathbb{k}[\sigma]$ is $\mathbb{Z}^{d_{\sigma}}$-graded, we also have the $\mathbb{Z}^{d_{\sigma}}$-graded version of a normalized dualizing complex

$$
\begin{aligned}
& { }^{*} D_{\sigma}^{\bullet}: 0 \longrightarrow \bigoplus_{\substack{\tau \leq \sigma, \operatorname{dim} \mathbb{k}[\tau]=d_{\sigma}}}{ }^{*} E_{\mathbb{k}[\sigma]}(\mathbb{k}[\tau]) \longrightarrow \bigoplus_{\substack{\tau \leq \sigma, \operatorname{dim} \mathbb{k}[\tau]=d_{\sigma}-1}}{ }^{*} E_{\mathbb{k}[\sigma]}(\mathbb{k}[\tau]) \longrightarrow \cdots \\
& \cdots \longrightarrow{ }^{*} E_{\mathbb{k}[\sigma]}(\mathbb{k}) \longrightarrow 0,
\end{aligned}
$$

where ${ }^{*} E_{\mathbb{k}[\sigma]}(-)$ denotes the injective hull in the category of $\mathbb{Z}^{d_{\sigma} \text {-graded }}$ $\mathbb{k}[\sigma]$-modules, and cohomological degrees are given by the same way as $D_{R}^{\bullet}$.

It is easy to see that the positive part

$$
\bigoplus_{a \in \mathbf{M}_{\sigma}}\left[{ }^{*} E_{\mathbb{k}[\sigma]}(\mathbb{k}[\tau])\right]_{a}
$$

of ${ }^{*} E_{\mathbb{k}[\sigma]}(\mathbb{k}[\tau])$ is isomorphic to $\mathbb{k}[\tau]$. Set

$$
I_{\sigma}^{\bullet}:=\bigoplus_{a \in \mathbf{M}_{\sigma}}\left[{ }^{*} D_{\sigma}^{\bullet}\right]_{a} \subset{ }^{*} D_{\sigma}^{\bullet} .
$$

Clearly, $I_{\sigma}^{\bullet}$ is a complex with

$$
I_{\sigma}^{i}:=\bigoplus_{\substack{\tau \leq \sigma, \operatorname{dim} \mathbb{k}[\tau]=-i}} \mathbb{k}[\tau] .
$$

As is well-known, $D_{\sigma}^{\bullet}$ is an injective resolution of ${ }^{*} D_{\sigma}^{\bullet}$ in the category $\operatorname{Mod}(\mathbb{k}[\sigma])$, and the latter can be seen as a subcomplex of the former in a non-canonical way. By the construction, $I_{\sigma}^{\bullet}$ is a subcomplex of ${ }^{*} D_{\sigma}^{\bullet}$, and $D_{\sigma}^{\bullet}$ is a subcomplex of $D_{R}^{\bullet}$. Combining them, we have an embedding $I_{\sigma}^{\bullet} \hookrightarrow D_{R}^{\bullet}$. Thus the problem is the compatibility of the embeddings $I_{\sigma}^{\bullet} \hookrightarrow D_{R}^{\bullet}$ and $I_{\tau}^{\bullet} \hookrightarrow D_{R}^{\bullet}$ for $\sigma, \tau \in \Sigma$.

Step 2. Canonical (up to scalar multiplication) embedding $\mathbb{k}[\sigma] \hookrightarrow D_{R}^{-d_{\sigma}}$.

For $\sigma \in \mathcal{X}$, let $\omega_{\mathbb{k}[\sigma]}$ be the canonical module of $\mathbb{k}[\sigma]$. By our hypothesis that $\mathcal{M}$ is cone-wise normal, we see that $\omega_{\mathbb{k}[\sigma]}$ is just the ideal generated by $\left\{t^{a} \in \mathbb{k}[\sigma] \mid a \in \operatorname{rel}-\operatorname{int}\left(C_{\sigma}\right) \cap \mathbf{M}_{\sigma}\right\}$ (cf. [4, Theorem 6.3.5]). Whence we have the exact sequence:

$$
0 \longrightarrow \omega_{\mathbb{k}}[\sigma] \longrightarrow \mathbb{k}[\sigma] \longrightarrow \mathbb{k}[\sigma] / \omega_{\mathbb{k}[\sigma]} \longrightarrow 0
$$


Since $\operatorname{Hom}_{R}\left(\mathbb{k}[\sigma] / \omega_{\mathbb{k}[\sigma]}, E_{R}(\mathbb{k}[\sigma])\right)=0$, applying $\operatorname{Hom}_{R}\left(-, E_{R}(\mathbb{k}[\sigma])\right)$ to the above exact sequence yields the canonical isomorphism

$$
\operatorname{Hom}_{R}\left(\mathbb{k}[\sigma], E_{R}(\mathbb{k}[\sigma])\right) \cong \operatorname{Hom}_{R}\left(\omega_{\mathbb{k}[\sigma]}, E_{R}(\mathbb{k}[\sigma])\right),
$$

and thus the canonical embedding

$$
\operatorname{Hom}_{R}\left(\omega_{\mathbb{k}[\sigma]}, E_{R}(\mathbb{k}[\sigma])\right) \cong\left\{x \in E_{R}(\mathbb{k}[\sigma]) \mid \mathfrak{p}_{\sigma} x=0\right\} \subset E_{R}(\mathbb{k}[\sigma]) .
$$

Since we have

$$
\begin{aligned}
\operatorname{Hom}_{R}\left(\omega_{\mathbb{k}[\sigma]}, D_{R}^{-d_{\sigma}}\right) & =\bigoplus_{\substack{\mathfrak{p} \in \operatorname{Spec} R \\
\operatorname{dim} R / \mathfrak{p}=d_{\sigma}}} \operatorname{Hom}_{R}\left(\omega_{\mathbb{k}[\sigma]}, E_{R}(R / \mathfrak{p})\right) \\
& =\operatorname{Hom}_{R}\left(\omega_{\mathbb{k}[\sigma]}, E_{R}(\mathbb{k}[\sigma])\right)
\end{aligned}
$$

in conjunction with (5.3), we obtain the canonical embedding

$$
\operatorname{Hom}_{R}\left(\omega_{\mathbb{k}[\sigma]}, D_{R}^{-d_{\sigma}}\right) \subset E_{R}(\mathbb{k}[\sigma]) \subset D_{R}^{-d_{\sigma}} .
$$

Since $\operatorname{Hom}_{R}\left(\omega_{\mathbb{k}[\sigma]}, D_{R}^{-d_{\sigma}-1}\right)=0$, it follows that

$$
\begin{aligned}
\operatorname{Ext}_{R}^{-d_{\sigma}}\left(\omega_{\mathbb{k}[\sigma]}, D_{R}^{\bullet}\right) & =\operatorname{Ker}\left(\operatorname{Hom}_{R}\left(\omega_{\mathbb{k}[\sigma]}, D_{R}^{-d_{\sigma}}\right) \rightarrow \operatorname{Hom}_{R}\left(\omega_{\mathbb{k}[\sigma]}, D_{R}^{-d_{\sigma}+1}\right)\right) \\
& =\left\{x \in D_{R}^{-d_{\sigma}} \mid \mathfrak{p}_{\sigma} x=0 \text { and } \partial\left(J_{\sigma} x\right)=0\right\},
\end{aligned}
$$

where $J_{\sigma}:=\left\{t^{a} \mid a \in \operatorname{rel}-\operatorname{int}\left(C_{\sigma}\right) \cap \mathbf{M}_{\sigma}\right\}$ and $\partial: D^{-d_{\sigma}} \rightarrow D^{-d_{\sigma}+1}$ is the differential map. Consequently, we have

$$
\mathbb{k}[\sigma] \cong \operatorname{Ext}_{R}^{-d_{\sigma}}\left(\omega_{\mathbb{k}[\sigma]}, D_{R}^{\bullet}\right) \subset D_{R}^{-d_{\sigma}}
$$

canonically.

Using this, we have a canonical injection

$$
I_{R}^{i}=\bigoplus_{\substack{\sigma \in \mathcal{X} \\ \operatorname{dim} \mathbb{k}[\sigma]=-i}} \mathbb{k}[\sigma] \longleftrightarrow D_{R}^{i}
$$

for each $i$.

Step 3. Compatibility.

For $\sigma, \tau \in \mathcal{X}$ with $\tau \leq \sigma$, set

$$
\underline{\operatorname{Ext}}_{\mathbb{k}[\sigma]}^{i}\left(\omega_{\mathbb{k}[\tau]},{ }^{*} D_{\sigma}^{\bullet}\right):=H^{i}\left(\operatorname{Hom}_{\mathbb{k}[\sigma]}^{\bullet}\left(\omega_{\mathbb{k}[\tau]},{ }^{*} D_{\sigma}^{\bullet}\right)\right) .
$$




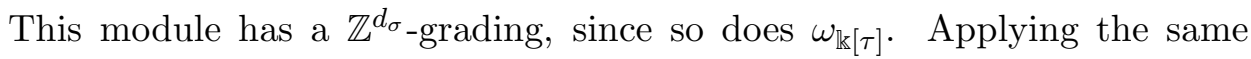
argument as in Step 2 (replacing $R$ by $\mathbb{k}[\sigma]$ and $D_{R}^{\bullet}$ by ${ }^{*} D_{\sigma}^{\bullet}$ ), we have a canonical embedding which is the first injection of the sequence

$$
\mathbb{k}[\tau] \cong \underline{\operatorname{Ext}}_{\mathbb{k}[\sigma]}^{-d_{\tau}}\left(\omega_{\mathbb{k}[\tau]},{ }^{*} D_{\sigma}^{\bullet}\right) \longleftrightarrow{ }^{*} D_{\sigma}^{-d_{\tau}} \longleftrightarrow D_{R}^{-d_{\tau}}
$$

Here the last injection is not canonical. Since the inclusions ${ }^{*} D_{\sigma}^{\bullet} \hookrightarrow D_{\sigma}^{\bullet} \hookrightarrow$ $D_{R}^{\bullet}$ give the isomorphisms

$$
\underline{\operatorname{Ext}}_{\mathbb{k}[\sigma]}^{-d_{\tau}}\left(\omega_{\mathbb{k}[\tau]},{ }^{*} D_{\sigma}^{\bullet}\right) \cong \operatorname{Ext}_{\mathbb{k}[\sigma]}^{-d_{\tau}}\left(\omega_{\mathbb{k}[\tau]}, D_{\sigma}^{\bullet}\right) \cong \operatorname{Ext}_{R}^{-d_{\tau}}\left(\omega_{\mathbb{k}[\tau]}, D_{R}^{\bullet}\right),
$$

the embedding $\mathbb{k}[\tau] \hookrightarrow D_{R}^{-d_{\tau}}$ given in (5.6) coincides with the one given in Step 2. (So the image of (5.6) does not depend on the choice of an injection ${ }^{*} D_{\sigma}^{-d_{\tau}} \hookrightarrow D_{R}^{-d_{\tau}}$.)

It is easy to see that the inclusion (5.1) (see also (5.2)) is same as the one given by (5.6). Therefore, through any ${ }^{*} D_{\sigma}^{\bullet} \hookrightarrow D_{R}^{\bullet}$, the embeddings of $(5.1)$ and (5.5) are compatible. So under this embedding, we have $I_{\sigma}^{i} \subset I_{R}^{i} \subset D_{R}^{i}$. Since $I_{\sigma}^{\bullet}$ is a subcomplex of $D_{R}^{\bullet}$ for all $\sigma \in \mathcal{X}, \bigoplus_{i \in \mathbb{Z}} I_{R}^{i}$ forms a subcomplex of $D_{R}^{\bullet}$.

We can take a generator $1_{\sigma} \in \mathbb{k}[\sigma] \subset I_{R}^{-d_{\sigma}} \subset D_{R}^{-d_{\sigma}}$ for each $\sigma \in \mathcal{X}$ satisfying

$$
\partial_{D_{R}^{\bullet}}\left(1_{\sigma}\right)=\sum \varepsilon^{\prime}(\sigma, \tau) \cdot 1_{\tau}
$$

for some incidence function $\varepsilon^{\prime}$ on $\mathcal{X}$. Recall that we have fixed an incidence function $\varepsilon$ to define the differential of $I_{R}^{\bullet}$. While $\varepsilon$ and $\varepsilon^{\prime}$ do not coincide in general, their difference is well-regulated (cf. [4, p. 265]). So, after a suitable change of $\left\{1_{\sigma}\right\}_{\sigma \in \mathcal{X}}$, we have

$$
\partial_{D_{R}^{\bullet}}\left(1_{\sigma}\right)=\sum \varepsilon(\sigma, \tau) \cdot 1_{\tau}
$$

Therefore we conclude that $I_{R}^{\bullet}$ is a subcomplex of $D_{R}^{\bullet}$ as is desired.

When $R$ is a normal semigroup ring, the second author showed in $[18$, Lemma 3.8] that there is a natural isomorphism between $\mathbb{D}$ and $\operatorname{RHom}\left(-, D_{R}^{\bullet}\right)$. The next result generalizes this to toric face rings.

Proposition 5.4. There is the following commutative diagram;

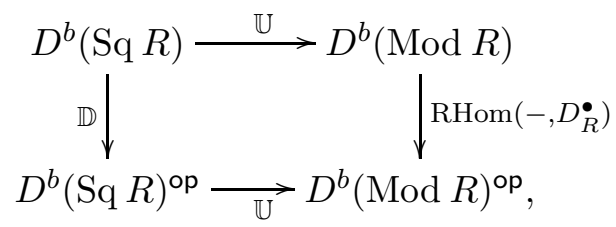


where $\mathbb{U}$ is the functor induced by the forgetful functor $\operatorname{Sq} R \rightarrow \operatorname{Mod} R$. In particular, we have $\mathbb{D}\left(M^{\bullet}\right) \cong \operatorname{RHom}_{R}\left(M^{\bullet}, D_{R}^{\bullet}\right)$ in $D^{b}(\operatorname{Mod} R)$ for any $M^{\bullet} \in D^{b}(\operatorname{Sq} R)$, and hence $\operatorname{Ext}_{R}^{i}\left(M^{\bullet}, D_{R}^{\bullet}\right)$ has a $\mathbb{Z} \mathcal{M}$-grading induced by $\mathbb{D}\left(M^{\bullet}\right)$.

Proof. Let Inj-Sq be the full subcategory of Sq $R$ consisting of all injective objects, that is, finite direct sums of $\mathbb{k}[\sigma]$ for various $\sigma \in \mathcal{X}$. As is wellknown (cf. [7, Proposition 4.7]), the bounded homotopy category $K^{b}$ (Inj-Sq) is equivalent to $D^{b}(\mathrm{Sq} R)$. It is easy to see that $\mathbb{D}(\mathbb{k}[\sigma])=\operatorname{Hom}_{R}^{\bullet}\left(\mathbb{k}[\sigma], I_{R}^{\bullet}\right)$. Moreover, $\mathbb{D}\left(J^{\bullet}\right)=\operatorname{Hom}_{R}^{\bullet}\left(J^{\bullet}, I_{R}^{\bullet}\right)$ for all $J^{\bullet} \in K^{b}($ Inj-Sq $)$. Since $I_{R}^{\bullet}$ is a subcomplex of $D_{R}^{\bullet}$ as shown in Proposition 5.3, we have a chain map $\operatorname{Hom}_{R}^{\bullet}\left(J^{\bullet}, I_{R}^{\bullet}\right) \rightarrow \operatorname{Hom}_{R}^{\bullet}\left(J^{\bullet}, D_{R}^{\bullet}\right)$. This map induces a natural transformation $\Psi: \mathbb{U} \circ \mathbb{D} \rightarrow \operatorname{RHom}_{R}\left(-, D_{R}^{\bullet}\right) \circ \mathbb{U}$. If $M \in \operatorname{Sq} R$ is a $\mathbb{k}[\sigma]$-module, then $\mathbb{D}(M) \cong \mathrm{RHom}_{\mathbb{k}[\sigma]}\left(M, D_{\sigma}^{\bullet}\right) \cong \operatorname{RHom}_{R}\left(M, D_{R}^{\bullet}\right)$ by [18, Lemma 3.8]. In particular, $\Psi(\mathbb{k}[\sigma])$ is isomorphism for all $\sigma \in \mathcal{X}$. Hence applying [7, Proposition 7.1], we see that $\Psi\left(M^{\bullet}\right)$ is an isomorphism for all $M^{\bullet} \in D^{b}(\operatorname{Sq} R)$.

The most part of the proof of Theorem 5.2 has done now.

Proof of Theorem 5.2. Since $R \in \mathrm{Sq} R$, we have

$$
I_{R}=\mathbb{D}(R) \cong \operatorname{RHom}_{R}\left(R, D_{R}^{\bullet}\right) \cong D_{R}^{\bullet}
$$

by Proposition 5.4 .

Let $M \in \operatorname{Mod}_{\mathbb{Z} \mathcal{M}} R$. We can construct the graded Matlis dual $M^{\vee} \in$ $\operatorname{Mod}_{\mathbb{Z} \mathcal{M}} R$ of $M$ as follows: For each $a \in|\mathbb{Z} \mathcal{M}|,\left(M^{\vee}\right)_{a}$ is the $\mathbb{k}$-dual space of $M_{-a}$. For $a, b \in|\mathbb{Z} \mathcal{M}|$ such that $a+b$ exists (that is, $a, b, a+b \in \mathbf{M}_{\sigma}$ for some $\sigma \in \mathcal{X})$, the multiplication map $\left(M^{\vee}\right)_{a} \ni x \mapsto t^{b} x \in\left(M^{\vee}\right)_{a+b}$ is the $\mathbb{k}$-dual of $M_{-a-b} \ni y \mapsto t^{b} y \in M_{-a}$. Otherwise, $t^{b} x=0$ for all $x \in\left(M^{\vee}\right)_{a}$.

It is obvious that $M^{\vee}$ is actually a $\mathbb{Z} \mathcal{M}$-graded $R$-module. If $\operatorname{dim}_{\mathbb{k}} M_{a}<$ $\infty$ for all $a \in|\mathbb{Z} \mathcal{M}|\left(\right.$ e.g. $M \in \bmod _{\mathbb{Z} \mathcal{M}} R$ ), then $M^{\vee \vee} \cong M$. Clearly, $(-)^{\vee}$ defines an exact contravariant functor from $\operatorname{Mod}_{\mathbb{Z} \mathcal{M}} R$ to itself. We can extend this functor to the functors $K^{b}\left(\operatorname{Mod}_{\mathbb{Z} \mathcal{M}} R\right) \rightarrow K^{b}\left(\operatorname{Mod}_{\mathbb{Z} \mathcal{M}} R\right)^{\text {op }}$ and $D^{b}\left(\operatorname{Mod}_{\mathbb{Z} \mathcal{M}} R\right) \rightarrow D^{b}\left(\operatorname{Mod}_{\mathbb{Z} \mathcal{M}} R\right)^{\mathrm{op}}$. We simply denote them by $(-)^{\vee}$.

Proposition 5.5. As functors from $D^{b}(\operatorname{Sq} R)$ to $D^{b}\left(\operatorname{Mod}_{\mathbb{Z} \mathcal{M}} R\right)$, we have $\mathrm{R} \Gamma_{\mathfrak{m}} \cong(-)^{\vee} \circ \mathbb{U} \circ \mathbb{D}$, where $\mathbb{U}: D^{b}(\operatorname{Sq} R) \rightarrow D^{b}\left(\operatorname{Mod}_{\mathbb{Z} \mathcal{M}} R\right)$ is induced by the forgetful functor $\mathrm{Sq} R \rightarrow \operatorname{Mod}_{\mathbb{Z} \mathcal{M}} R$. In particular, if $M \in \mathrm{Sq} R$, then $H_{\mathfrak{m}}^{i}(M) \cong \operatorname{Ext}_{R}^{-i}\left(M, D_{R}^{\bullet}\right)^{\vee}$ as $\mathbb{Z} \mathcal{M}$-graded modules for all $i$. 
Proof. We use the notation of the proofs of the above results. If $M \in$ $\operatorname{Mod}_{\mathbb{Z} \mathcal{M}} R$, then the $|\mathcal{M}|$-graded part $\bigoplus_{a \in|\mathcal{M}|} M_{a}$ of $M$ is clearly an $R$ submodule. For $\tau \in \Sigma$, recall that $T_{\tau}=\left\{t^{a} \mid a \in \mathbf{M}_{\tau}\right\}$ is a multiplicatively closed set. It is easy to see that, for $\sigma, \tau \in \Sigma$, the localization $T_{\tau}^{-1} \mathbb{k}[\sigma]$ is non-zero if and only if $\tau \leq \sigma$. When $\tau \leq \sigma$, the $|\mathcal{M}|$-graded part of $\left(T_{\tau}^{-1} \mathbb{k}[\sigma]\right)^{\vee}$ is isomorphic to $\mathbb{k}[\tau]$.

Let $L_{R}^{\bullet}$ be the Céch complex of $R$ defined in Section 3. It is easy to see that the $|\mathcal{M}|$-graded part of $\left(L_{R}^{\bullet} \otimes_{R} \mathbb{k}[\sigma]\right)^{\vee}$ is isomorphic to $\mathbb{D}(\mathbb{k}[\sigma])$. Moreover, if $J^{\bullet} \in K^{b}(\operatorname{Inj}-\mathrm{Sq})$, then the $|\mathcal{M}|$-graded part of $\left(L_{R}^{\bullet} \otimes_{R} J^{\bullet}\right)^{\vee}$ is isomorphic to $\mathbb{D}\left(J^{\bullet}\right)$. Thus $\mathbb{D}\left(J^{\bullet}\right)$ is a subcomplex of $\left(L_{R}^{\bullet} \otimes_{R} J^{\bullet}\right)^{\vee}$, and there is a chain map $L_{R}^{\bullet} \otimes_{R} J^{\bullet} \rightarrow \mathbb{D}\left(J^{\bullet}\right)^{\vee}$. Recall that $L_{R}^{\bullet} \otimes_{R} J^{\bullet}$ is quasi-isomorphic to $R \Gamma_{\mathfrak{m}}\left(J^{\bullet}\right)$ by Corollary 3.3. Hence we have a natural transformation $\Phi: R \Gamma_{\mathfrak{m}} \rightarrow(-)^{\vee} \circ \mathbb{U} \circ \mathbb{D}$, where we regard $\mathrm{R} \Gamma_{\mathfrak{m}}$ and $(-)^{\vee} \circ \mathbb{U} \circ \mathbb{D}$ as functors from $K^{b}(\operatorname{Inj}-\mathrm{Sq})\left(\cong D^{b}(\operatorname{Sq} R)\right)$ to $D^{b}\left(\operatorname{Mod}_{\mathbb{Z} \mathcal{M}} R\right)$. Since $\Phi(\mathbb{k}[\sigma])$ is an isomorphism for all $\sigma \in \mathcal{X}, \Phi$ is a natural isomorphism by $[7$, Proposition 7.1].

\section{$\S 6 . \quad$ Sheaves associated with squarefree modules}

Throughout this section, $\mathcal{M}$ is a cone-wise normal monoidal complex supported by a conical complex $(\Sigma, \mathcal{X})$. Recall that $X=\bigcup_{\sigma \in \mathcal{X}} \sigma$ is the underlying topological space of the cell complex $\mathcal{X}$. As in the previous section, let $\Lambda$ be the incidence algebra of the poset $\mathcal{X}$ over $\mathbb{k}$, and $\bmod \Lambda$ the category of finitely generated left $\Lambda$-modules.

Let $\operatorname{Sh}(X)$ be the category of sheaves of finite dimensional $\mathbb{k}$-vector spaces on $X$. We say $\mathcal{F} \in \operatorname{Sh}(X)$ is constructible with respect to the cell decomposition $\mathcal{X}$, if the restriction $\left.\mathcal{F}\right|_{\sigma}$ is a constant sheaf for all $\emptyset \neq \sigma \in \mathcal{X}$.

In [17], the second author constructed the functor $(-)^{\dagger}: \bmod \Lambda \rightarrow$ $\operatorname{Sh}(X)$. (Under the convention that $\emptyset \notin \mathcal{X}$, this functor has been wellknown to specialists.) Here we give a precise construction for the reader's convenience.

For $M \in \bmod \Lambda$, set

$$
\operatorname{Spé}(M):=\bigcup_{\emptyset \neq \sigma \in \mathcal{X}} \sigma \times M_{\sigma} .
$$

Let $\pi: \operatorname{Spé}(M) \rightarrow X$ be the projection map which sends $(p, m) \in \sigma \times$ $M_{\sigma} \subset \operatorname{Spé}(M)$ to $p \in \sigma \subset X$. For an open subset $U \subset X$ and a map $s: U \rightarrow \operatorname{Spé}(M)$, we will consider the following conditions: 
(*) $\pi \circ s=\operatorname{id}_{U}$ and $s_{p}=e_{\sigma, \tau} \cdot s_{q}$ for all $p \in \sigma \cap U, q \in \tau \cap U$ with $\sigma \geq \tau$. Here $s_{p}$ (resp. $\left.s_{q}\right)$ is the element of $M_{\sigma}\left(\operatorname{resp} . M_{\tau}\right)$ with $s(p)=\left(p, s_{p}\right)$ (resp. $\left.s(q)=\left(q, s_{q}\right)\right)$.

(**) There is an open covering $U=\bigcup_{i \in I} U_{i}$ such that the restriction of $s$ to $U_{i}$ satisfies $(*)$ for all $i \in I$.

Now we define a sheaf $M^{\dagger} \in \operatorname{Sh}(X)$ from $M$ as follows. For an open set $U \subset X$, set

$$
M^{\dagger}(U):=\{s \mid s: U \rightarrow \operatorname{Spé}(M) \text { is a map satisfying }(* *)\}
$$

and the restriction map $M^{\dagger}(U) \rightarrow M^{\dagger}(V)$ is the natural one. It is easy to see that $M^{\dagger}$ is a constructible sheaf with respect to the cell decomposition $\mathcal{X}$. For $\sigma \in \mathcal{X}$, let $U_{\sigma}:=\bigcup_{\tau \geq \sigma} \tau$ be an open set of $X$. Then we have $M^{\dagger}\left(U_{\sigma}\right) \cong$ $M_{\sigma}$. Moreover, if $\sigma \leq \tau$, then we have $U_{\sigma} \supset U_{\tau}$ and the restriction map $M^{\dagger}\left(U_{\sigma}\right) \rightarrow M^{\dagger}\left(U_{\tau}\right)$ corresponds to the multiplication map $M_{\sigma} \ni x \mapsto$ $e_{\tau, \sigma} x \in M_{\tau}$. For a point $p \in \sigma$, the stalk $\left(M^{\dagger}\right)_{p}$ of $M^{\dagger}$ at $p$ is isomorphic to $M_{\sigma}$. This construction gives the exact functor $(-)^{\dagger}: \bmod \Lambda \rightarrow \operatorname{Sh}(X)$. We also remark that $M_{\emptyset}$ is irrelevant to $M^{\dagger}$.

As in the previous sections, let $R=\mathbb{k}[\mathcal{M}]$ be the toric face ring, and Sq $R$ the category of squarefree $R$-modules. Through the equivalence Sq $R \cong$ $\bmod \Lambda,(-)^{\dagger}: \bmod \Lambda \rightarrow \operatorname{Sh}(X)$ gives the exact functor

$$
(-)^{+}: \operatorname{Sq} R \longrightarrow \operatorname{Sh}(X)
$$

Recall that $X$ admits Verdier's dualizing complex $\mathcal{D}_{X}^{\bullet} \in D^{b}(\operatorname{Sh}(X))$ with coefficients in $\mathbb{k}$ (see $[10, \mathrm{~V}$. Section 2]). In [17], the second author considered the duality functor $\mathbf{D}: D^{b}(\bmod \Lambda) \rightarrow D^{b}(\bmod \Lambda)$. Through the functor $(-)^{\dagger}: \bmod \Lambda \rightarrow \operatorname{Sh}(X)$, D corresponds to Poincaré-Verdier duality on $D^{b}(\operatorname{Sh}(X))$. More precisely, [17, Theorem 3.2] states that, for $M^{\bullet} \in D^{b}(\bmod \Lambda)$, we have

$$
\mathbf{D}\left(M^{\bullet}\right)^{\dagger} \cong \mathrm{R} \mathcal{H} \operatorname{om}\left(\left(M^{\bullet}\right)^{\dagger}, \mathcal{D}_{X}^{\bullet}\right)
$$

in $D^{b}(\operatorname{Sh}(X))$. On the other hand, through the equivalence $\bmod \Lambda \cong \operatorname{Sq} R$, the duality $\mathbf{D}$ on $D^{b}(\bmod \Lambda)$ corresponds to our duality $\mathbb{D}$ on $D^{b}(\operatorname{Sq} R)$ up to translation. More precisely, $\mathbb{D}(-)[-1]$ corresponds to $\mathbf{D}(-)$, where the complex $M^{\bullet}[-1]$ of a complex $M^{\bullet}$ denotes the degree shifting of $M^{\bullet}$ with $M^{\bullet}[-1]^{i}=M^{i-1}$. So we have the following. 
Theorem 6.1. For $M^{\bullet} \in D^{b}(\mathrm{Sq} R)$, we have

$$
\mathbb{D}\left(M^{\bullet}\right)^{+}[-1] \cong \operatorname{RHom}\left(\left(M^{\bullet}\right)^{+}, \mathcal{D}_{X}^{\bullet}\right)
$$

in $D^{b}(\operatorname{Sh}(X))$. In particular, $\left(I_{R}^{\bullet}\right)^{+}[-1] \cong \mathcal{D}_{X}^{\bullet}$, where $I_{R}^{\bullet}$ is the complex constructed in the previous section.

By Proposition 5.5, if $M \in \mathrm{Sq} R$, then we have

$$
H_{\mathfrak{m}}^{i}(M)^{\vee} \cong \operatorname{Ext}_{R}^{-i}\left(M, D_{R}^{\bullet}\right) \in \mathrm{Sq} R .
$$

Hence $H_{\mathfrak{m}}^{i}(M)$ is $-|\mathcal{M}|$-graded and the next result determines the "Hilbert function" of $H_{\mathfrak{m}}^{i}(M)$.

TheOREm 6.2. If $M \in \mathrm{Sq} R$, we have the following.

(a) There is an isomorphism

$$
H^{i}\left(X, M^{+}\right) \cong\left[H_{\mathfrak{m}}^{i+1}(M)\right]_{0} \quad \text { for all } i \geq 1,
$$

and an exact sequence

$$
0 \longrightarrow\left[H_{\mathfrak{m}}^{0}(M)\right]_{0} \longrightarrow M_{0} \longrightarrow H^{0}\left(X, M^{+}\right) \longrightarrow\left[H_{\mathfrak{m}}^{1}(M)\right]_{0} \longrightarrow 0 .
$$

(b) If $0 \neq a \in|\mathcal{M}|$ with $\sigma=\operatorname{supp}(a)$, then

$$
\left[H_{\mathfrak{m}}^{i}(M)\right]_{-a} \cong H_{c}^{i-1}\left(U_{\sigma},\left.M^{+}\right|_{U_{\sigma}}\right)
$$

for all $i \geq 0$. Here $U_{\sigma}=\bigcup_{\tau \geq \sigma} \tau$ is an open set of $X$, and $H_{c}^{\bullet}(-)$ stands for the cohomology with compact support.

Proof. (a) We have $H^{i}(\mathbb{D}(M)) \cong \operatorname{Ext}_{R}^{i}\left(M, D_{R}^{\bullet}\right) \cong H_{\mathfrak{m}}^{-i}(M)^{\vee}$ by Proposition 5.5. On the other hand, via the equivalence $\mathrm{Sq} R \cong \bmod \Lambda, \mathbb{D}(-)[-1]$ corresponds to the duality $\mathbf{D}(-)=\operatorname{RHom}_{\Lambda}\left(-, \omega^{\bullet}\right)$ of $D^{b}(\bmod \Lambda)$ introduced in [17]. So the assertion follows from [17, Corollary 3.5, Theorem 2.2].

(b) Similarly, it follows from [17, Lemma 5.1].

In the sequel, $\tilde{H}^{i}(X ; \mathbb{k})$ denotes the $i^{\text {th }}$ reduced cohomology of $X$ with coefficients in $\mathbb{k}$. That is, $\tilde{H}^{i}(X ; \mathbb{k}) \cong H^{i}(X ; \mathbb{k})$ for all $i \geq 1$, and $\tilde{H}^{0}(X ; \mathbb{k}) \oplus$ $\mathbb{k} \cong H^{0}(X ; \mathbb{k})$. Here $H^{i}(X ; \mathbb{k})$ is the usual cohomology of $X$ with coefficients in $\mathbb{k}$. 
Corollary 6.3. (cf. Brun et al. [1, Theorem 1.3]) With the above notation, we have $\left[H_{\mathfrak{m}}^{i}(R)\right]_{0} \cong \tilde{H}^{i-1}(X ; \mathbb{k})$ and $\left[H_{\mathfrak{m}}^{i}(R)\right]_{-a} \cong H_{c}^{i-1}\left(U_{\sigma}, \underline{\mathbb{k}}_{U_{\sigma}}\right)$ for all $i \geq 0$ and all $0 \neq a \in|\mathcal{M}|$. Here $\sigma=\operatorname{supp}(a)$, and $\underline{\mathbb{k}}_{U_{\sigma}}$ is the $\mathbb{k}$-constant sheaf on $U_{\sigma}$.

Proof. The second isomorphism is a direct consequence of Theorem 6.2 (b) and the fact that $R^{+} \cong \underline{\mathbb{k}}_{X}$. So it suffices to show the first. By the isomorphism of Theorem $6.2(\mathrm{a}),\left[H_{\mathfrak{m}}^{i}(R)\right]_{0} \cong H^{i-1}\left(X, R^{+}\right) \cong H^{i-1}\left(X, \underline{\mathbb{k}}_{X}\right) \cong$ $H^{i-1}(X ; \mathbb{k}) \cong \tilde{H}^{i-1}(X ; \mathbb{k})$ for all $i \geq 2$. Similarly, by the exact sequence of the theorem and that $H_{\mathfrak{m}}^{0}(R)=0$, we have $0 \rightarrow R_{0} \rightarrow H^{0}(X ; \mathbb{k}) \rightarrow$ $\left[H_{\mathfrak{m}}^{1}(R)\right]_{0} \rightarrow 0$. Since $R_{0}=\mathbb{k}$, we have $\left[H_{\mathfrak{m}}^{1}(R)\right]_{0} \cong \tilde{H}^{0}(X ; \mathbb{k})$.

We say $R$ is a Buchsbaum ring, if $R_{\mathfrak{m}^{\prime}}$ is a Buchsbaum local ring for all maximal ideal $\mathfrak{m}^{\prime}$. See [13] for further information.

Theorem 6.4. Set $\operatorname{dim} X=d$ (equivalently, $\operatorname{dim} R=d+1$ ). Then $R$ is Buchsbaum if and only if $\mathcal{H}^{i}\left(\mathcal{D}_{X}^{\bullet}\right)=0$ for all $i \neq-d$. In particular, the Buchsbaum property of $R$ is a topological property of $X$ (while it might depend on $\operatorname{char}(\mathbb{k}))$.

Proof. Assume that $\mathcal{H}^{i}\left(\mathcal{D}_{X}^{\bullet}\right) \neq 0$ for some $i \neq-d$ (equivalently, $-d+$ $1 \leq i \leq 0)$. Then $\left[H^{i-1}\left(I_{R}^{\bullet}\right)\right]_{a} \neq 0$ for some $0 \neq a \in|\mathcal{M}|$ by Theorem 6.1 . Since $H^{i-1}\left(I_{R}^{\bullet}\right)$ is squarefree, we have $\operatorname{dim}_{\mathbb{k}}\left(H^{i-1}\left(I_{R}^{\bullet}\right) \otimes_{R} R_{\mathfrak{m}}\right)=\infty$. Since $H^{i-1}\left(I_{R}^{\bullet}\right) \otimes_{R} R_{\mathfrak{m}}$ is the Matlis dual of $H_{\mathfrak{m}}^{1-i}\left(R_{\mathfrak{m}}\right)$ over the local ring $R_{\mathfrak{m}}$, we have $\operatorname{dim}_{\mathbb{k}} H_{\mathfrak{m}}^{1-i}\left(R_{\mathfrak{m}}\right)=\infty$ and $R_{\mathfrak{m}}$ is not Buchsbaum.

Conversely, assume that $\mathcal{H}^{i}\left(\mathcal{D}_{X}^{\bullet}\right)=0$ for all $i \neq-d$. Then $H^{i}\left(I_{R}^{\bullet}\right)=$ $\left[H^{i}\left(I_{R}^{\bullet}\right)\right]_{0}$ for all $i \neq-d-1$, and they are $\mathbb{k}$-vector spaces (that is, $R / \mathfrak{m}$ modules). Hence $H^{i}\left(I_{R}^{\bullet}\right) \otimes_{R} R_{\mathfrak{m}^{\prime}}=0$ for all $i \neq-d-1$ and all $\mathfrak{m}^{\prime}$ with $\mathfrak{m}^{\prime} \neq \mathfrak{m}$. Thus $R_{\mathfrak{m}^{\prime}}$ is Cohen-Macaulay (in particular, Buchsbaum). It remains to show that $R_{\mathfrak{m}}$ is Buchsbaum. Set $T^{\bullet}:=\tau_{-d-1} I_{R}^{\bullet}$. Here, for a complex $M^{\bullet}$ and an integer $r, \tau_{-r} M^{\bullet}$ denotes the truncated complex

$$
\cdots \longrightarrow 0 \longrightarrow \operatorname{Im}\left(M^{-r} \rightarrow M^{-r+1}\right) \longrightarrow M^{-r+1} \longrightarrow M^{-r+2} \longrightarrow \cdots .
$$

By the assumption, we have $H^{i}\left(T^{\bullet}\right)=\left[H^{i}\left(T^{\bullet}\right)\right]_{0}$ for all $i$. Since $T^{\bullet}$ is a complex of $\mathcal{M}$-graded modules, $U^{\bullet}:=\bigoplus_{0 \neq a \in|\mathcal{M}|}\left(T^{\bullet}\right)_{a}$ is a subcomplex of $T^{\bullet}$, and a natural map $T^{\bullet} \rightarrow\left(T^{\bullet} / U^{\bullet}\right)$ is a quasi-isomorphism by the above observation. Since $T^{\bullet} / U^{\bullet}$ is a complex of $\mathbb{k}$-vector spaces, $R_{\mathfrak{m}}$ is Buchsbaum by $[13$, II.Theorem 4.1]. 
If $\operatorname{dim} X=d$ and $R$ is Buchsbaum, we set r $_{X}:=\mathcal{H}^{-d}\left(\mathcal{D}_{X}^{\bullet}\right) \in \operatorname{Sh}(X)$. The next fact follows from [10, IX, (4.1)].

Proposition 6.5. (Poincaré duality) With the above situation, we have $H^{i}(X ; \mathbb{k}) \cong H^{d-i}\left(X\right.$, or $\left._{X}\right)$ for all $i$.

If $X$ is a $d$-dimensional manifold (with or without boundary), then $R$ is Buchsbaum and $o r_{X}$ is the usual orientation sheaf of $X$ with coefficients in $\mathbb{k}$ (see, for example, [10, III, §8]). When $X$ is an orientable manifold, then or ${ }_{X} \cong \underline{\mathbb{k}}_{X}$. In this case, Proposition 6.5 is nothing other than the classical Poincaré duality.

Assume that $\operatorname{dim} X=d$, equivalently, $\operatorname{dim} R=d+1$. If $R$ is Buchsbaum, we call $\omega_{R}:=H^{-d-1}\left(I_{R}^{\bullet}\right) \in \mathrm{Sq} R$ the canonical module of $R$. Clearly, $\left(\omega_{R}\right)^{+} \cong o r_{X}$

EXAMPLE 6.6. Recall the toric face ring $R$ given in Example 2.9, whose underlying topological space $X$ is the Möbius strip. Clearly, $X$ is a manifold with boundary and $R$ is Buchsbaum. It is easy to see that $\tilde{H}^{2}(X ; \mathbb{k})=0$ and or $_{X} \cong i_{\text {! }} \underline{\mathbb{k}}_{X \backslash \partial X}$, where $\underline{\mathbb{k}}_{X \backslash \partial X}$ is the $\mathbb{k}_{\text {-constant sheaf on } X \backslash \partial X(\partial X \text { denotes }}$ the boundary of $X$ ), and $i: X \backslash \partial X \hookrightarrow X$ is the embedding map. Hence the canonical module $\omega_{R}$ is isomorphic to the monomial ideal $I$ with $I^{+} \cong$ $i_{!} \underline{\mathbb{k}}_{X \backslash \partial X}$. So we have $\omega_{R} \cong\left(X_{x} X_{u}, X_{z} X_{w}, X_{v} X_{y}, X_{x} X_{z}, X_{y} X_{w}, X_{x} X_{v}\right)$, where the right side is an ideal of $R$.

We say $R$ is Gorenstein*, if it is Cohen-Macaulay and $\omega_{R} \cong R$ as $\mathbb{Z} \mathcal{M}$ graded modules.

TheOrem 6.7. Set $d:=\operatorname{dim} X$.

(a) (Caijun, [6]) $R$ is Cohen-Macaulay if and only if $\mathcal{H}^{i}\left(\mathcal{D}_{X}^{\bullet}\right)=0$ for all $i \neq-d$, and $\tilde{H}^{i}(X ; \mathbb{k})=0$ for all $i \neq d$.

(b) Assume that $d \geq 1$ and $R$ is Cohen-Macaulay. Then $R$ is Gorenstein*, if and only if or $_{X} \cong \mathbb{k}_{X}$, if and only if $\left(\text { or }_{X}\right)_{p} \cong \mathbb{k}$ for all $p \in X$ and $H^{d}(X ; \mathbb{k}) \neq 0$. Here $\underline{\mathbb{k}}_{X}$ denotes the $\mathbb{k}$-constant sheaf on $X$ and $\left(\text { or }_{X}\right)_{p}$ is the stalk of the sheaf or $X$ at $p$.

Proof. (a) Since $\operatorname{dim} R=d+1, R$ is Cohen-Macaulay if and only if $H^{i}\left(I_{R}^{\bullet}\right)\left(=\operatorname{Ext}_{R}^{i}\left(R, D_{R}^{\bullet}\right)\right)=0$ for all $i \neq-d-1$. By Theorem 6.1 , the above conditions are also equivalent to that $\mathcal{H}^{i}\left(\mathcal{D}_{X}^{\bullet}\right)=0$ for all $i \neq-d$ 
and $\left[H^{i}\left(I_{R}^{\bullet}\right)\right]_{0}=0$ for all $i \neq-d-1$. Since $\left[H^{i}\left(I_{R}^{\bullet}\right)\right]_{0} \cong\left(\left[H_{\mathfrak{m}}^{-i}(R)\right]_{0}\right)^{*} \cong$ $\tilde{H}^{-i-1}(X ; \mathbb{k})^{*}$, we are done.

(b) We show the first equivalence. If $R$ is Gorenstein*, then or $_{X} \cong$ $\left(\omega_{R}\right)^{+} \cong R^{+} \cong \underline{\mathbb{k}}_{X}$. So we get the necessity. Next assume that or $_{X}(=$ $\left.\left(\omega_{R}\right)^{+}\right) \cong \underline{\mathbb{k}}_{X}$. Then we have that

$$
\left[\omega_{R}\right]_{a}=\mathbb{k} \quad \text { for all } 0 \neq a \in|\mathcal{M}| .
$$

On the other hand, by Proposition 6.5 , we have $\left[\omega_{R}\right]_{0}^{\vee} \cong\left[H_{\mathfrak{m}}^{d+1}(R)\right]_{0} \cong$ $H^{d}(X ; \mathbb{k}) \cong H^{0}\left(X\right.$, or $\left._{X}\right) \cong H^{0}(X ; \mathbb{k}) \cong \mathbb{k}$ ( since $R$ is Cohen-Macaulay and $d \geq 1, \tilde{H}^{0}(X ; \mathbb{k})=0$ and $X$ is connected). Take a non-zero element $x \in\left[\omega_{R}\right]_{0}$. Since $\omega_{R}$ is a squarefree $R$-module, $M:=R x$ is a squarefree submodule of $\omega_{R}$. Set

$$
\begin{aligned}
\Upsilon & :=\left\{\operatorname{supp}(a)|a \in| \mathcal{M} \mid, M_{a}=\left[\omega_{R}\right]_{a}\right\} \\
& =\left\{\operatorname{supp}(a)|a \in| \mathcal{M} \mid, M_{a} \neq 0\right\} \subset \mathcal{X} .
\end{aligned}
$$

Here the second equality follows from the condition (6.1). It is easy to see that $\sigma \leq \tau \in \Upsilon$ implies $\sigma \in \Upsilon$. So we have a direct sum decomposition $\omega_{R}=M \oplus\left(\bigoplus_{\operatorname{supp}(a) \in|\mathcal{M}| \backslash \Upsilon}\left[\omega_{R}\right]_{a}\right)$ as an $R$-module. On the other hand, $\omega_{R}$ is indecomposable. Hence $\omega_{R}=M \cong R$ as $\mathbb{Z} \mathcal{M}$-graded modules. So we get the sufficiency.

For the second equivalence, it is enough to prove the sufficiency. Since $\left[\omega_{R}\right]_{0} \cong H^{d}(X ; \mathbb{k}) \neq 0$, we can take $0 \neq x \in\left[\omega_{R}\right]_{0}$. By argument similar to the above, $(R x)^{+}$is a direct summand of or $_{X}$. Note that $X$ is connected and $\underline{\mathbb{k}}_{X}$ is indecomposable. Since $\underline{\mathbb{k}}_{X} \cong \mathcal{E} x t^{-d}\left(\right.$ or $\left._{X}, \mathcal{D}_{X}^{\bullet}\right)$, or ${ }_{X}$ is also indecomposable. Hence $o r_{X} \cong(R x)^{+} \cong \underline{\mathbb{k}}_{X}$. We are done.

COROLlary 6.8. The Cohen-Macaulay property and Gorenstein* property of $R$ are topological properties of $X$ (while it may depend on $\operatorname{char}(\mathbb{k})$ ).

Proof. Most of the statement is a direct consequence of Theorems 6.7. It remains to consider the Gorenstein* property in the case $\operatorname{dim} R=0$. Then $R$ is Gorenstein* if and only if $X$ consists of exactly two points. So the assertion is clear.

Remark 6.9. The main result of Caijun [6] is much more general than our Theorems 6.7 (a). However, since he worked in a wider context, his argument does not give precise information of local cohomologies and canonical modules. 
Recall that $\mathcal{M}$ admits a finite subset $\left\{a_{e}\right\}_{e \in E}$ of $|\mathcal{M}|$ generating $\mathbb{k}[\mathcal{M}]$ as a $\mathbb{k}$-algebra. Then the polynomial $\operatorname{ring} S:=\mathbb{k}\left[X_{e} \mid e \in E\right]$ surjects on $\mathbb{k}[\mathcal{M}]$. Let $I_{\mathcal{M}}$ be its kernel (i.e., $\left.\mathbb{k}[\mathcal{M}]=S / I_{\mathcal{M}}\right)$. A remarkable result $[5$, Theorem 3.8] of Bruns et al. shows that (if $\mathcal{M}$ is cone-wise normal) there is a generating set $\left\{a_{e}\right\}_{e \in E}$ and a term order $\succ$ on $S$ such that the initial ideal $\operatorname{in}_{\succ}\left(I_{\mathcal{M}}\right)$ is a radical monomial ideal. In this case, $\operatorname{in}_{\succ}\left(I_{\mathcal{M}}\right)$ equals to the Stanley-Reisner ring $I_{\Delta}$ of a simplicial complex $\Delta$ which gives a triangulation of $X$. Hence, by a basic fact on Gröbner bases, the sufficiency of Theorems 6.4 and 6.7 (b) follow from their result, at least under the additional assumption that $R$ admits an $\mathbb{N}$-grading with $R_{0}=\mathbb{k}$.

\section{REFERENCES}

[1] M. Brun, W. Bruns and T. Römer, Cohomology of partially ordered sets and local cohomology of section rings, Adv. Math., 208 (2007), 210-235.

[2] W. Bruns and J. Gubeladze, Polyhedral algebras, arrangements of toric varieties, and their groups, Computational commutative algebra and combinatorics, Adv. Stud. Pure Math., 33, 2001, pp. 1-51.

[3] W. Bruns and J. Gubeladze, Polytopes, rings, and $K$-theory, Springer Monographs in Mathematics, Springer, 2009.

[4] W. Bruns and J. Herzog, Cohen-Macaulay rings, revised edition, Cambridge University Press, 1998.

[5] W. Bruns, R. Koch, and T. Römer, Gröbner bases and Betti numbers of monoidal complexes, Michigan Math. J., 57 (2008), 71-91.

[6] Z. Caijun, Cohen-Macaulay section rings, Trans. Amer. Math. Soc., 349 (1997), 4659-4667.

[7] R. Hartshorne, Residues and duality, Lecture notes in Mathematics 20, Springer, 1966.

[8] B. Ichim and T. Römer, On toric face rings, J. Pure Appl. Algebra, 210 (2007), 249-266.

[9] M.-N. Ishida, The local cohomology groups of an affine semigroup ring, Algebraic Geometry and Commutative Algebra, vol. I, Kinokuniya, Tokyo, 1988, pp. 141-153.

[10] B. Iversen, Cohomology of sheaves, Springer-Verlag, 1986.

[11] R. Y. Sharp, Dualizing complexes for commutative Noetherian rings, Math. Proc. Comb. Phil. Soc., 78 (1975), 369-386.

[12] R. P. Stanley, Generalized H-vectors, intersection cohomology of toric varieties, and related results, Commutative algebra and combinatorics, Adv. Stud. Pure Math., 11, 1987, 187-213.

[13] S. Stuckrad and W. Vogel, Buchsbaum rings and applications, Springer-Verlag, 1986.

[14] K. Yanagawa, Sheaves on finite posets and modules over normal semigroup rings, J. Pure Appl. Algebra, 161 (2001), 341-366. 
[15] K. Yanagawa, Squarefree modules and local cohomology modules at monomial ideals, Local cohomology and its applications, Lecture Notes in Pure and Appl. Math., 226, Dekker, New York, 2002, pp. 207-231.

[16] K. Yanagawa, Stanley-Reisner rings, sheaves, and Poincaré-Verdier duality, Math. Res. Lett., 10 (2003), 635-650.

[17] K. Yanagawa, Dualizing complex of the incidence algebra of a finite regular cell complex, Illinois J. Math., 49 (2005), 1221-1243.

[18] K. Yanagawa, Notes on C-graded modules over an affine semigroup ring $K[C]$, Comm. Algebra, 38 (2008), 3122-3146.

[19] G. Ziegler, Lectures on Polytopes, Graduate Texts in Mathematics, Vol. 152, Springer, 1995 (Revised edition 1998).

Ryota Okazaki

Department of Pure and Applied Mathematics

Graduate School of Information Science and Technology

Osaka University

Toyonaka, Osaka, 560-0043

Japan

u574021d@ecs.cmc.osaka-u.ac.jp

Kohji Yanagawa

Department of Mathematics

Faculty of Engineering Science

Kansai University

Suita, 564-8680

Japan

yanagawa@ipcku.kansai-u.ac.jp 